\title{
3-Dimensional Time-Domain Full-Wave Analysis of Optical Array Antennas
}

\author{
Benedikt Oswald ${ }^{1}, *$, Aleksejs Fomins ${ }^{2}$, Arya Fallahi ${ }^{1}$, Patrick Leidenberger ${ }^{3}$, and Peter Bastian ${ }^{4}$ \\ ${ }^{1}$ Paul Scherrer Institut (PSI), GFA, CH-5232 Villigen \\ ${ }^{2}$ School of Physics and Astronomy, University of St Andrews, Fife KY16 9AX, Scotland \\ ${ }^{3}$ Swiss Federal Institute of Technology Zurich, Laboratory for Electromagnetic Fields and Microwave Electronics (IFH), CH-8092 Zurich \\ ${ }^{4}$ Heidelberg University, Interdisciplinary Center for Scientific Computing (IWR) D-69120 Heidelberg, Germany
}

\begin{abstract}
We report on the 3-dimensional full-wave analysis of optical array antennas that employ a dipole element as the fundamental building block. We use a finite element time domain (FETD) method discretized on unstructured tetrahedral grids in order to efficiently resolve the geometry which has a wide range of characteristic length scales; from the nanometer to the micrometer range. Such devices are useful in a number of applications in order to convert propagating electromagnetic energy into localized energy which is concentrated within a spot whose dimension is significantly smaller than the wavelength. This capability is especially useful for field emitter arrays (FEA) used in novel, ultra-low emittance photocathodes. The antenna elements are modeled with gold metallic properties in the optical region of the electromagnetic spectrum. There, gold is a dispersive dielectric material and desribed with a Drude dielectric material model. To support the validity of our analysis we numerically analyze electromagnetic problems that can be solved analytically, thus benchmarking the algorithm. We then computationally analyze a single dipole element in free space and a logarithmically periodic array of dipoles, similar to the concept of the Yagi-Uda array antenna in the microwave region. We demonstrate the existence of resonant modes on the dipole rod elements. Eventually, we comment on further development work.
\end{abstract}

Keywords: Array Antennas, Sub-Wavelength Size, Dispersive Dielectric Model, Drude Model Computational Electrodynamics, Electromagnetic, Field Emitter Arrays (FEA), Finite Element Time Domain Method, FETD, Optical Antennas.

\section{INTRODUCTION}

The concept of laser induced field emission from nanometer structured metal tips ${ }^{11,44,45}$ arranged on a regular grid, aka. field emitter arrays (FEA), has got considerable technological potential for the fabrication of ultra-low emittance electron sources ${ }^{14,19,23,41,48,49}$ in a wide range of application fields, such as photocathodes for accelerators, e.g., the X-ray free electron laser ${ }^{12}$ project SwissFEL (http://www.psi.ch/swissfel) currently under construction at the Paul Scherrer Institut (PSI), ultra-fast electron and single-electron microscopy and displays. ${ }^{22}$ Conceptually, the fundamental building block is a pyramidally shaped molybdenum tip ${ }^{23,48,49}$ which is illuminated by incoming laser light. The FEA represent a nano-optical problem: (1) because the wavelength of the incoming laser pulse is much larger than the dimensions of the field emitter (2) because the spot of field-enhancement around the tips must be considerably smaller than half of the wavelength.

${ }^{*}$ Author to whom correspondence should be addressed.
An electromagnetic wave can not be focused onto a spot smaller than about half the wavelength; this is generally known as the diffraction limit ${ }^{10}$ or, Abbe's limit or the Rayleigh criterion. The current that can be extracted from a single tip, according to the Fowler-Nordheim model ${ }^{13}$ depends on the local electric field. The emitted beam shall have ultra-low emittance, thus we want to minimize the area from where electrons are emitted. Thus, the lateral dimensions of a single field emitter array is on the order of a few nanometers only. ${ }^{23}$ Then, conflicting requirements result: (i) we need to focus the incoming laser light onto a spot size that corresponds to the emission area which clearly violates the diffraction limit and (ii) we need to maximize the electric field strength on the emission area. In this case, the laser wavelength $\lambda$ is on the order of a few hundred nanometers. In order to circumvent this, near-field optical techniques have been developed over the last 30 years, e.g.,. ${ }^{17,25,32,33,37}$ These techniques rely on the near-by probing of light scattered from an object where the distance between the scatterer and the probe is on the order of a few nanometers only. 
Thus, the distance is considerably smaller than the wavelength. More recently, optical antennas ${ }^{1,8,9,21,28-30,39}$ have therefore become attractive concepts to design nanometerstructured field-emission cathodes to focus incoming laser light onto a spot that is significantly smaller than half the wavelength. The principal idea of the optical antenna ${ }^{8}$ is to design a device that efficiently converts free-propagating optical radiation to localized energy and localized energy to propagating radiation. When applied to a nanometer structured field emitter array tip this implies to create an electromagnetic 'hot-spot' in the immediate vicinity of the sharp metallic tip. ${ }^{36,40,42}$ The diameter of the 'hot-spot' is then on the order of the tip's lateral dimensions. Thus, we obtain a significantly smaller 'hot-spot,' achieve electric field enhancement and eventually succeed in increasing the current extracted from the tip via field emission. In the case of laser induced field emission the static acceleration voltage is augmented by the time dependent electric field of the incoming laser light. Also, simply increasing laser power will not work since the field emitter element will eventually be destroyed either thermally or through discharge. Thus, we need to optimize the effect of given static acceleration voltage and the laser light collected in the region where we wish electron emission to occur. On the other hand, there are regions where the tip should not emit electrons. In other words, we must devise a structure in the vicinity of the tip with such an effect that it maximizes the electric field at the tip and minimizes it elsewhere. Thus, we need a focusing and directing structure. Optical antennas are devices designed exactly for this purpose. While the simpler designs employ metal, often gold, nano-spheres or nano-rods ${ }^{21,24,27,30}$ more complicated structures have been studied as well., ${ }^{2,47}$ In particular Mohammadi et al. ${ }^{27}$ employed a sophisticated body of revolution (BOR) approach to model the 3-dimensional shapes while Taminiau et al. ${ }^{47}$ analyzed an optical YagiUda antenna in 3-dimensional space but at one single frequency only. In this study we explore the capability of the finite element time domain (FETD) method $^{20,34-36,51}$ for modeling complicated nano-optical problems, optical antennas in particular, that employ metallic structures.

\section{FORMULATION OF THE PROBLEM}

Here, we numerically analyze optical dipole antennas with finite geometrical dimensions in the time domain, without assuming any symmetry. The fact of finite dimensions is emphasized here because the canonical analysis of dipole antennas almost always assumes infinitely thin metallic rods. $^{3,52}$ In particular, we analyze 2 different configurations, Figure 13, namely:

(1) a single dipole in free space, consisting of two separate arms made from gold; the dipole arm is modeled with a cylinder, capped by a hemi-spherical shape; the radius of the cylinder equals the radius of the hemi-spherical cap; the 2 dipole arms are separated by a short gap, $w_{\text {gap }}=$ $10 \mathrm{~nm}$, in between them; this elementary dipole serves as the fundamental building block for the electromagnetic analysis.

(2) an array of dipole antennas, consisting of a driving dipole, a reflector dipole and 3 director dipoles; the director dipoles become longer the further away they are from the driver dipole; in particular, the director dipoles' lengths are logarithmically-periodic (log-per), since the length of director 2 is 1.5 times the length of director 1 and director 3 is 1.5 times as long as director 2. Thus, this arrangement is related to the Yagi-Uda antenna, well known from microwave applications ${ }^{3}$ where it is often used as television (TV) aerial due to its highly directive radiation pattern; however, in the original Yagi-Uda design the dipole elements become shorter the further away they are from the director. We have inverted the scaling law in order to study what happens if we continuously reduce the lengths of the dipoles towards the location of the gap between the driver dipole arms.

In both cases, the antenna is illuminated with an incoming transverse-electric-magnetic (TEM) wave that models the illuminating laser light. The shape of the time dependence is chosen to be a sine carrier modulated by a Neumann pulse. We numerically calculate the 3-dimensional electromagnetic field distribution for the 2 different dipole antenna arrangements, case (i) and (ii). The simulation parameters for both cases are given in Table III. The array dipoles are made from gold, which in the visible range of the electromagnetic spectrum is best modeled with a dispersive dielectric material law. Here, we use the Drude model. ${ }^{32}$ We comment that the Drude model becomes less accurate w.r.t. experimental data towards shorter wavelengths..$^{38,50}$ Therefore, it is often extended by a Lorentz model ${ }^{50}$ in order to better reproduce the imaginary part of the complex dielectric permittivity. In this study we may restrict ourselves to the Drude model since we wish to demonstrate the 3-dimensional analysis of complicated metallic structures in the time domain, without resorting to symmetry or reducing the problem to 2 dimensions. Thus, the emphasis is on the general capability to model metals realistically with dispersive dielectrics in the time domain and less on specific material properties. We also study the enhancement of the electric field in the gap between the driving dipole's arms and the capability of the arrangement to collect incoming electromagnetic power from the laser.

\section{METHODS}

\subsection{The Finite Element Time Domain Method}

We use the computational electrodynamics code HADES3D $\mathrm{D}^{34-36}$ based on the FETD method ${ }^{20,51}$ for the 
Table I. Model parameters of the Hertzian dipole benchmark calculations. We particularly mention that the time signal shape has been shifted to the right in order to obtain a smooth excitation of the system; this generally applies to the analyzed configurations.

\begin{tabular}{|c|c|c|c|c|c|}
\hline Model resolution & Small & Medium & Large & Very large & {$[-]$} \\
\hline \multicolumn{6}{|c|}{ Geometry of computational domain } \\
\hline Shape & & & ere & & {$[-]$} \\
\hline \multirow{2}{*}{\multicolumn{6}{|c|}{$\begin{array}{c}1 \\
\text { Discretization parameters }\end{array}$}} \\
\hline & & & & & \\
\hline Mesh size [\#tetrahedra] & $24^{\prime} 968$ & $199^{\prime} 744$ & $1^{\prime} 597^{\prime} 952$ & $12^{\prime} 783^{\prime} 616$ & {$[-]$} \\
\hline Time step $\Delta t$ & 10 & 5 & 2.5 & 1.0 & [ps] \\
\hline Number timesteps & $2^{\prime} 000$ & $4^{\prime} 000$ & $8^{\prime} 000$ & $20^{\prime} 000$ & {$[-]$} \\
\hline \multicolumn{6}{|c|}{ Hertzian dipole parameters } \\
\hline Time signal shape & \multicolumn{4}{|c|}{ Neumann pulse ( $1^{\text {st }}$ derivative of Gaussian) } & {$[-]$} \\
\hline Excitation width $\sigma$ & \multicolumn{4}{|c|}{1.0} & {$[\mathrm{~ns}]$} \\
\hline Excitation offset $t_{0}$ & \multicolumn{4}{|c|}{10.0} & [ns] \\
\hline \multicolumn{6}{|c|}{ Computational resources } \\
\hline Number of cores used & 8 & 8 & 64 & 512 & {$[-]$} \\
\hline
\end{tabular}

3-dimensional full-wave analysis of optical antenna configurations. The HADES3D code solves the electric field vector wave, aka. curl-curl Eq. (1) in 3-dimensional space and the time domain on unstructured tetrahedral grids. The expression $\mathscr{L}(\mathbf{E})$ represents an operator used for modeling dispersive dielectric material

$$
\nabla \times \frac{1}{\mu} \nabla \times \mathbf{E}+\sigma \frac{\partial}{\partial t} \mathbf{E}+\epsilon_{0} \epsilon_{\infty}^{\prime} \frac{\partial^{2} \mathbf{E}}{\partial^{2} t}+\mathscr{L}(\mathbf{E})=-\frac{\partial}{\partial t} \mathbf{J}:(1) 25.180 .202 \underset{\text { Rat, }}{\partial t} \operatorname{Lug} 201204: 38: 23 \text { Drude }(\mathbf{E}) \stackrel{\text { def }}{=} \epsilon_{0} \omega_{p}^{2}\left(1-\gamma_{p} U(t) *\right) \mathbf{E}
$$
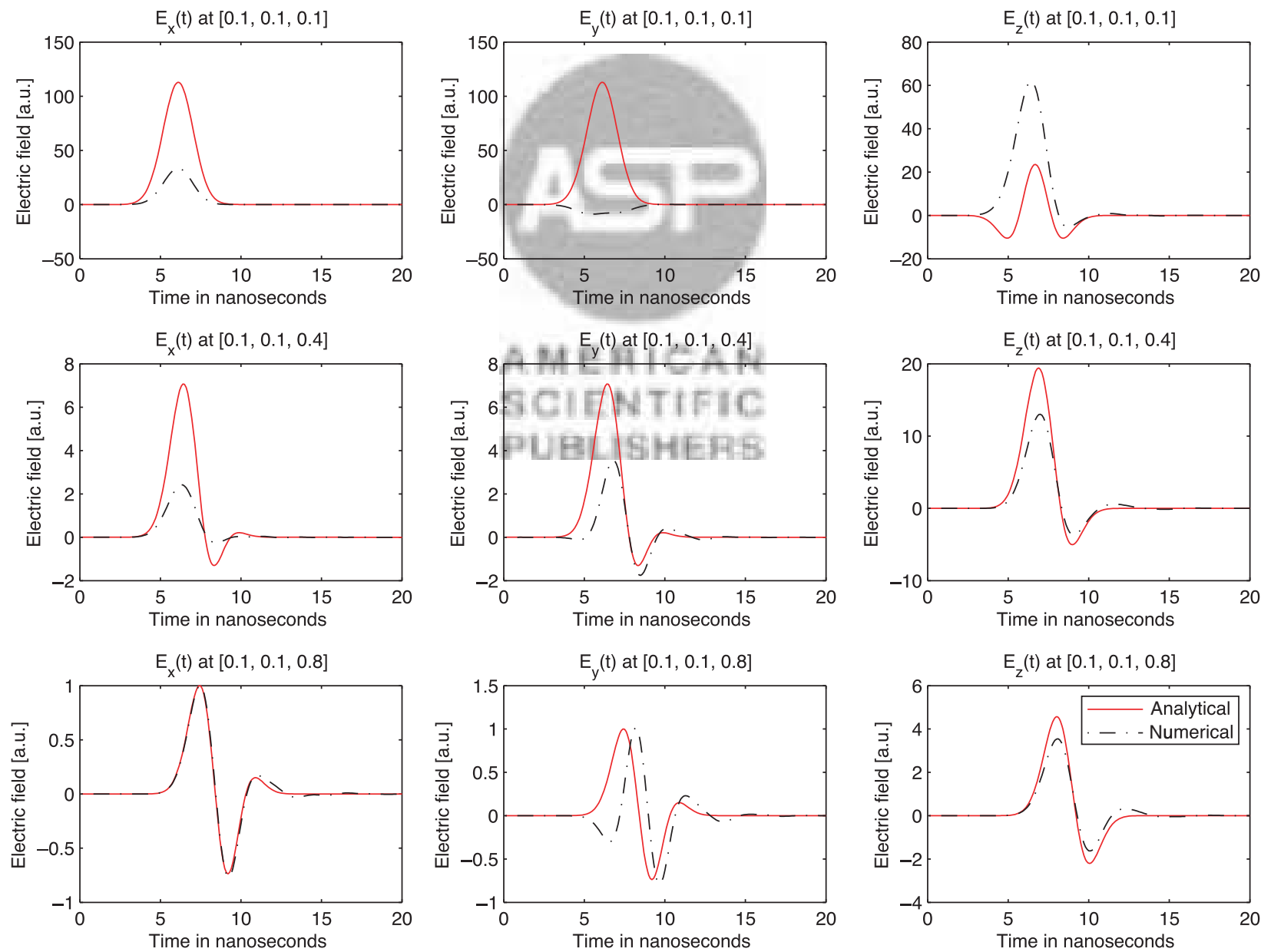

Fig. 1. Plot parameters: mesh size small, i.e. $24^{\prime} 968$ tetrahedra, cf. first column of Table (I) and $1^{\text {st }}$ order basis functions: we calculate the electric field $\mathbf{E}(\mathbf{x}, t)$ radiated by a Hertzian dipole that is situated at the center of the spherical computational domain with HADES3D and compare the cartesian components of the field vector against the analytical solution in the time domain. 
for the Drude model where $*$ denotes the convolution operator and $U(t)$ the Heaviside unit step function, respectively. The details of this derivation are given in a previous paper. ${ }^{34}$ Specifically, using a tetrahedral mesh offers substantial advantages when curvilinear geometry is discretized since it avoids the staircasing approximation that plagues the finite difference time domain (FDTD) method $^{46}$ which can only discretize curved geometry on an orthogonal cartesian grid. In the FDTD method the width of the cells essentially depends on the smallest geometrical details and hence leads to vast inefficiencies when large volumes must be discretized with a very small cell size. In contrast, in the FETD method the tetrahedral discretization of complicated geometry allows for level of detail modeling (LoD) so that a fine mesh is created where fine, physically relevant details must be resolved and coarser elements are generated elsewhere. We use the piecewise-linear-recursive-convolution (PLRC) method $^{46}$ for efficiently modeling dispersive dielectric materials in the time domain. In particular, we use the
Drude parameters for gold given by Novotny and Hecht. ${ }^{32}$ After discretizing the curl-curl Eq. (1) in space we obtain a system of linear ordinary differential equations (ODE) that are, still, continuous in time. We then use the central difference scheme for the temporal discretization ${ }^{20}$ which results in a linear system of Eq. (3) that must be solved to advance in time

$$
\mathrm{A} \mathbf{x}=\mathbf{b}
$$

where $\mathbf{x}$ represents the unknown vector of degrees of freedom at the next time step. The central difference scheme is conditionally stable and requires a timestep that is smaller than a certain limit. ${ }^{20}$ We particularly comment that the matrix A does not contain the discretized curl-curl vector operator from Eq. (1). This avoids problems due to the illconditioned nature of this matrix. Additionally, we comment that this scheme is sometimes denoted as explicit, in contrast to an implicit scheme that includes the discretized curl-curl operator. This should not be confused with the notion of explicit when it is used in the context of the FDTD method. ${ }^{46}$ There, it is meant to denote
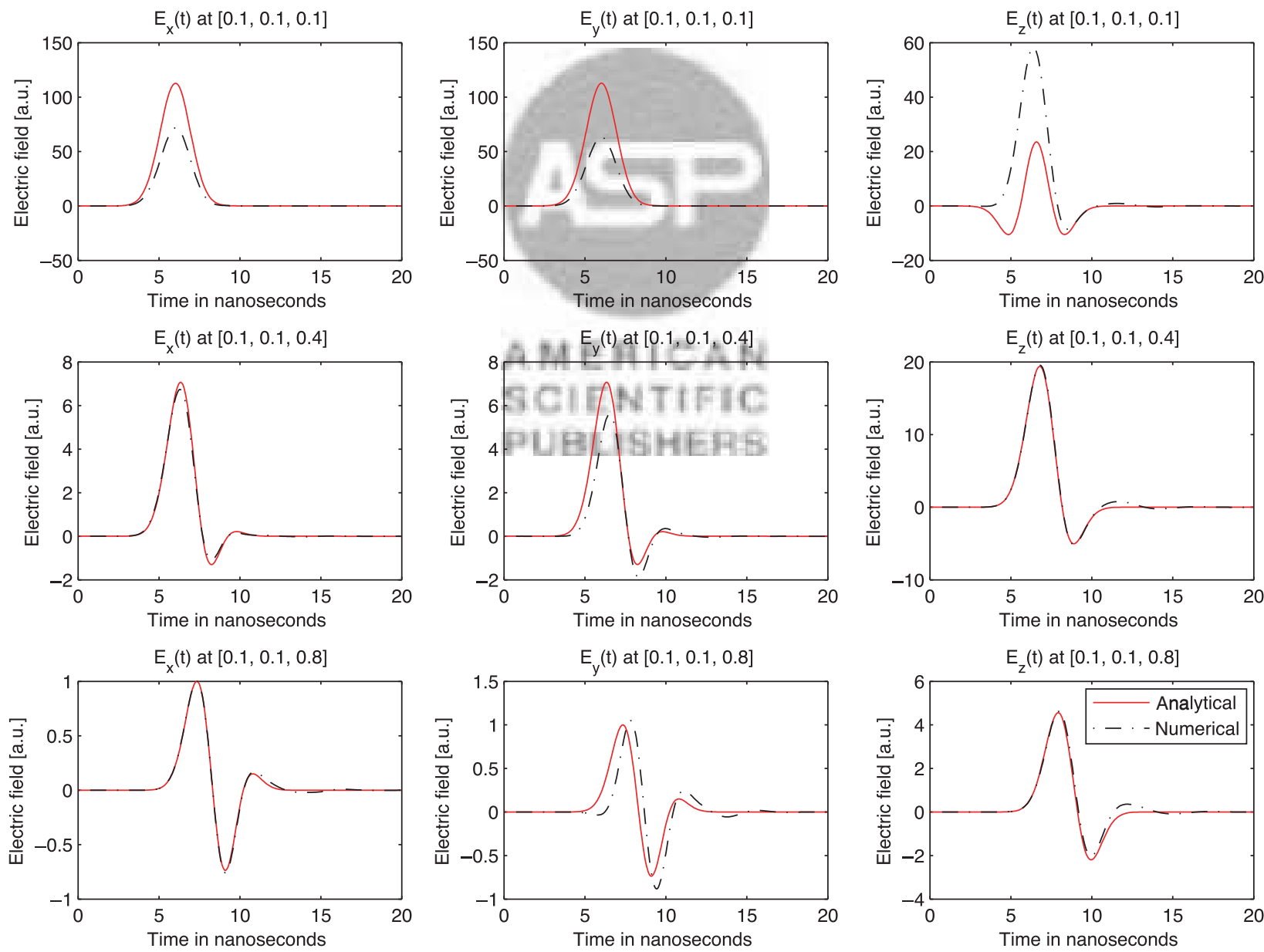

Fig. 2. Plot parameters: mesh size medium, i.e. $199^{\prime} 744$ tetrahedra, cf. second column of Table (I) and $1^{\text {st }}$ order basis functions: we calculate the electric field $\mathbf{E}(\mathbf{x}, t)$ radiated by a Hertzian dipole that is situated at the center of the spherical computational domain with HADES3D and compare the cartesian components of the field vector against the analytical solution in the time domain. 
a scheme that uses an elaborate sequence of comparably simple multiply-and-add operations to advance in time and, therefore, does not require a linear system to be solved. Here, we use the term explicit w.r.t. the partial matrices employed in the derivation of the global system matrix $A$. In our algorithm, the system matrix $A$ is large and highly sparse and its size directly depends both on the number of tetrahedral elements and the order, i.e., number, of the employed basis functions, used for approximating the electric field. Since the linear system must be solved at every time step, in order to advance in time, accelerating the linear system solution will reduce calculation time considerably. In today's computing landscape this inevitably leads to parallelization. The HADES3D code has thus been implemented so that it profits from the widely available distributed memory parallel compute clusters, based on the message passing interface (MPI) ${ }^{16}$ and the Dune library. ${ }^{6,7}$ Consequentially, the HADES3D code can be used from a modern single core laptop computer up to the massively parallel Cray XT5 (www.cray.com) and other supercomputers.

\subsection{Higher Order Basis Functions}

In the finite element method the unknown quantity, in our study this is the electric field vector, is approximated with functions of known shape but yet unknown scaling coefficients, aka. DoF. The DoF are obtained from the solution of the linear system of Eq. (3) at every timestep. The more rapidly the electric field varies, the more important it becomes to use flexible basis functions well suited to approximate the electric field. The most simple approach uses linear edge basis functions of the Whitney type..$^{20,51}$ The accuracy of computational results is then increased through refining the tetrahedral mesh by subdividing every tetrahedron into 8 new, smaller-size tetrahedra. While this approach may eventually converge to quite accurate solutions it is certainly not the most efficient one. ${ }^{20}$ Faster convergence is achieved if higher order basis functions are employed for approximating the electric field and if the solution is regular enough. That is generally the case for Maxwell's equations in our application field. In particular, exponential convergence is possible. ${ }^{20}$ In Section 3.4
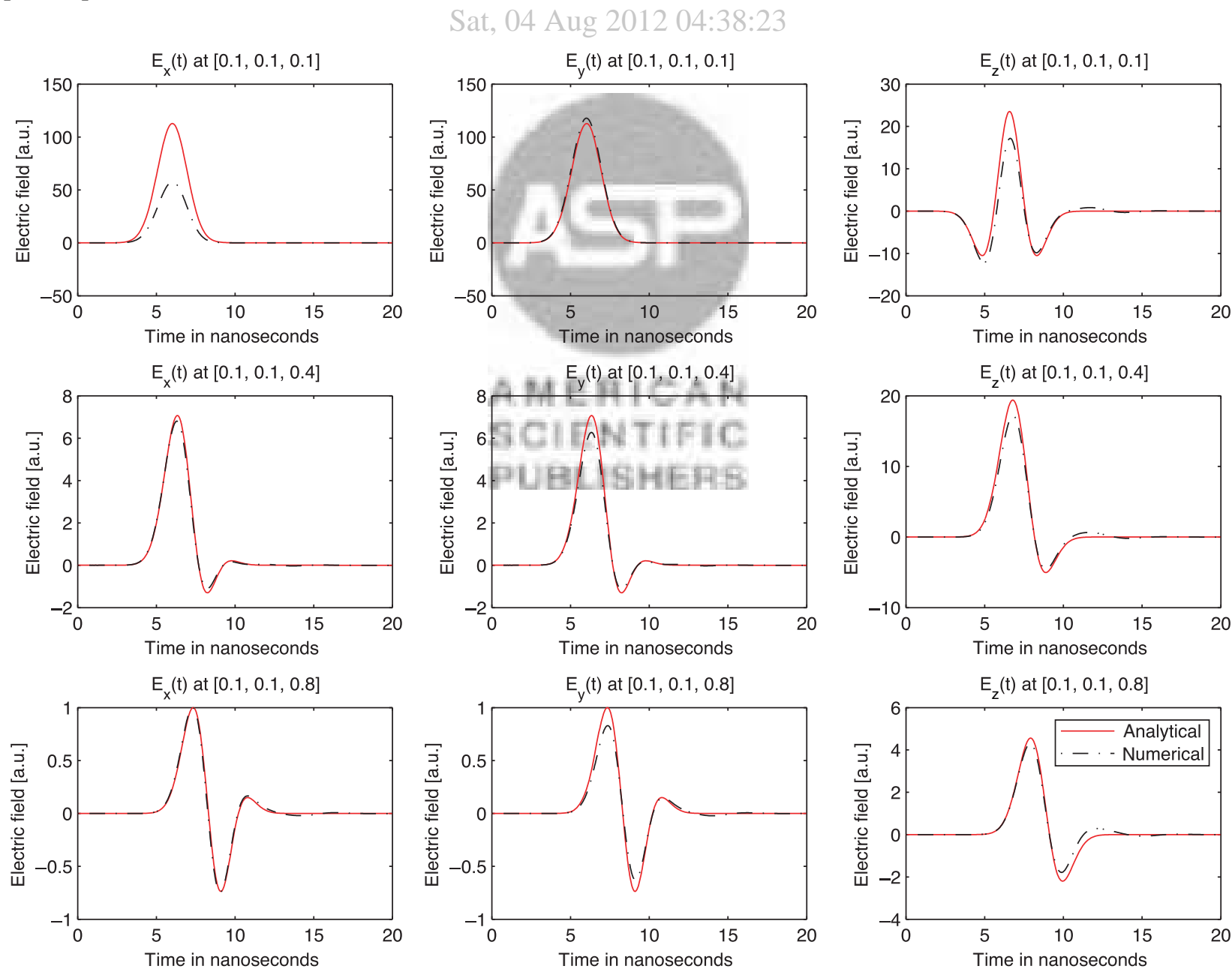

Fig. 3. Plot parameters: mesh size large, i.e. $1^{\prime} 597^{\prime} 952$ tetrahedra, cf. third column of Table (I) and $1^{\text {st }}$ order basis functions: we calculate the electric field $\mathbf{E}(\mathbf{x}, t)$ radiated by a Hertzian dipole that is situated at the center of the spherical computational domain with HADES3D and compare the cartesian components of the field vector against the analytical solution in the time domain. 
we show that even a coarse mesh in combination with higher order basis functions leads to more accurate solutions than a multiply refined mesh that employs only linear basis functions. As a somewhat premature conclusion we state that, whenever possible, higher order basis functions should be employed since they provide better accuracy at dramatically reduced computational cost. On the other hand, we comment that using higher order basis functions tends to increase the condition number of the global matrix and thus, inevitably, leads to an increased number of iterations, and then takes more time to solve the system.

\subsection{Parallel Linear Solver}

In general, large, sparse linear systems of equations, especially if they result from an explicit time discretization, are best solved using iterative linear solvers ${ }^{20,43}$ since direct methods, based on Gaussian elimination, can not cope with systems of such size within acceptable calculation time. ${ }^{15}$ The iterative approach seeks the solution to the system $A \mathbf{x}=\mathbf{b}$ through a successive improvement of an initial vector $\mathbf{x}_{0}$ towards a more accurate solution $\mathbf{x}_{n}$ until the solution vector is deemed to be accurate enough. This accuracy criterion is denoted as convergence and usually defined through the residual error, aka. defect vector $\mathbf{d}_{n}=$ A $\mathbf{x}_{n}-\mathbf{b}$. Once, $\mathbf{d}$ has become small enough, the iterative process has converged and it is terminated. The various iterative algorithms mainly differ in the method how the successive approximations $\mathbf{x}_{n}, \mathbf{x}_{n+1}$ to the exact solution are calculated and, hence, in the number of iterations required until the iterative process has converged. The next approximation $\mathbf{x}_{n+1}$ to the solution is computed from the previous iteration value $\mathbf{x}_{n}$ through the addition of a functional evaluation of the correction vector $\mathbf{v}$. The number of iterations required, until convergence has been reached, also sensitively depends on the condition of the matrix which in turn depends on the quality of the mesh; the number of iterations further depends on the order of the basis functions and the material properties. In contrast to stationary problems solved with the finite element method or discretized implicitly in time, the condition of the matrix does not depend on the size of the elements of the mesh. ${ }^{4}$ Here, we use an iterative solver based on the conjugate gradient (CG) method. ${ }^{20,43}$ To accelerate the solution of
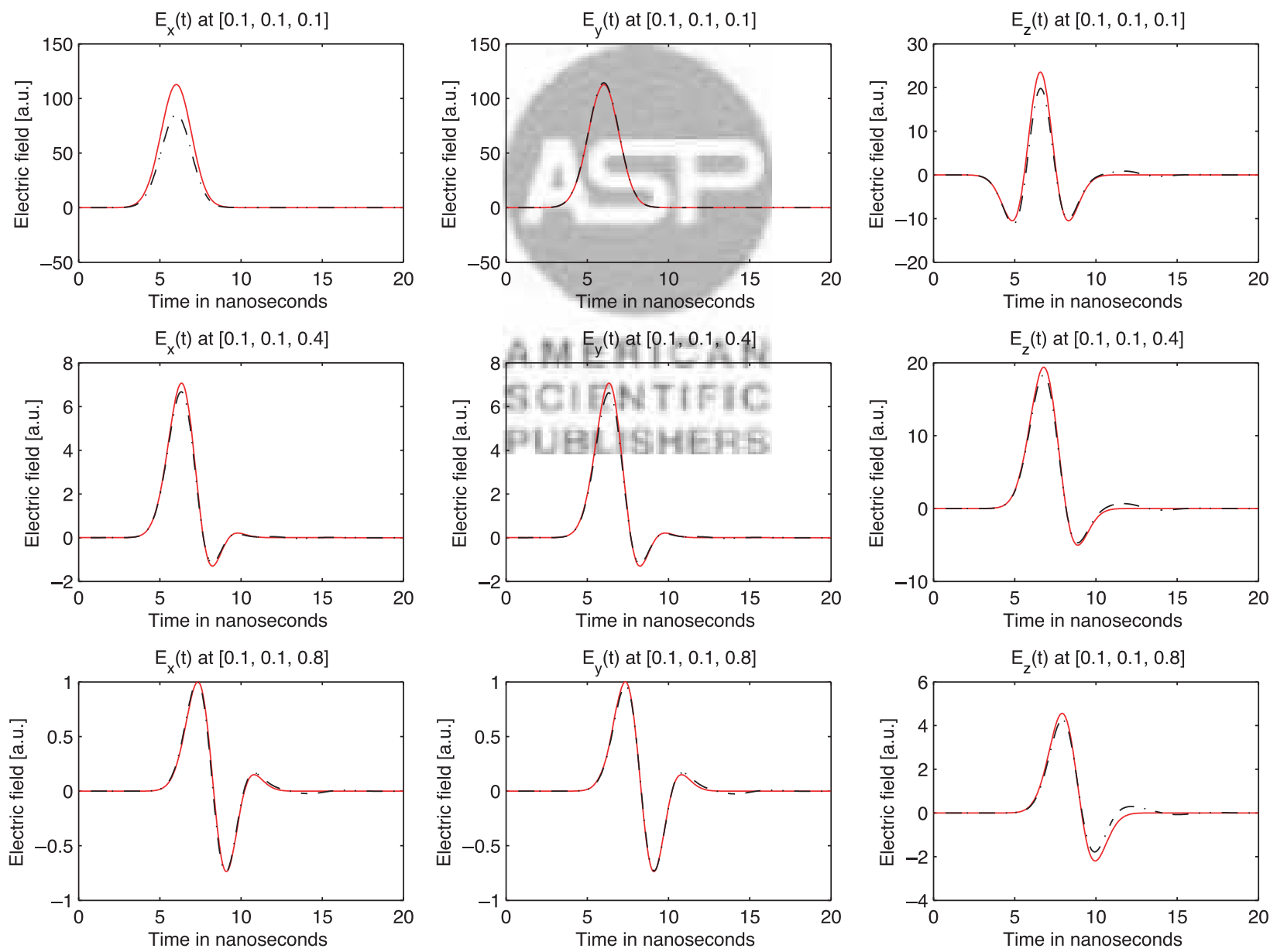

Fig. 4. Plot parameters: mesh size very large, i.e. $12^{\prime} 783^{\prime} 616$ tetrahedra, cf. fourth column of Table (I) and $1^{\text {st }}$ order basis functions: we calculate the electric field $\mathbf{E}(\mathbf{x}, t)$ radiated by a Hertzian dipole that is situated at the center of the spherical computational domain with HADES3D and compare the cartesian components of the field vector against the analytical solution in the time domain. 
A $\mathbf{x}=\mathbf{b}$ we employ preconditioning. ${ }^{51}$ Therefore, we leftmultiply the system with an approximation to the inverse of the original matrix $A$.

$$
\mathbf{v}_{n}^{\prime}=\mathrm{C}^{-1} \mathbf{d}=\mathrm{C}^{-1} \mathrm{~A} \mathbf{x}_{n}-\mathrm{C}^{-1} \mathbf{b}
$$

In order to accelerate the solution process and to reduce the number of iterations we have implemented a nonoverlapping parallel Jacobi preconditioner ${ }^{43}$ which uses the inverted diagonal part of $A$ as the approximation to its inverse

$$
c_{i j}=\delta_{i j} \cdot a_{i j}
$$

We then calculate the product of the Jacobi preconditioning matrix $\mathrm{C}^{-1}$ and the preconditioned defect vector $\mathbf{d}^{\prime}$ and pass it on to the CG solver, implemented in the Dune library. ${ }^{5}$ We mention that the deployment of a Jacobi preconditioner reduces the number of iterations required by a
Richardson preconditioner, where the $\mathbf{v}$ vector is identical to the defect vector $\mathbf{d}$, by a factor of 30 or even more. We also note that we have used the Dune library on a parallel, distributed memory cluster computer. In particular, we have implemented the parallel Jacobi preconditioner via the collective communication methods provided by Dune.

\subsection{Benchmark Calculations}

In order to assess performance and accuracy of the HADES3D code we have numerically analyzed electromagnetic problems that have analytical solutions and therefore serve as rigorous benchmarks, namely:

(1) the Hertzian dipole radiating into free space; and (2) scattering off a dielectric sphere made from gold ${ }^{26}$ with dielectric properties described by non-dispersive or Drude $^{32}$ dispersive dielectric properties.

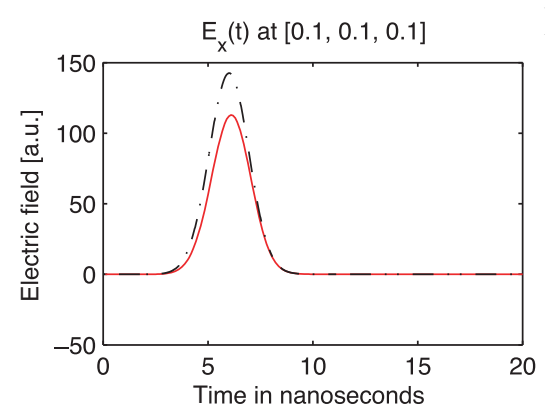

\section{Delivered by Ingenta to:}
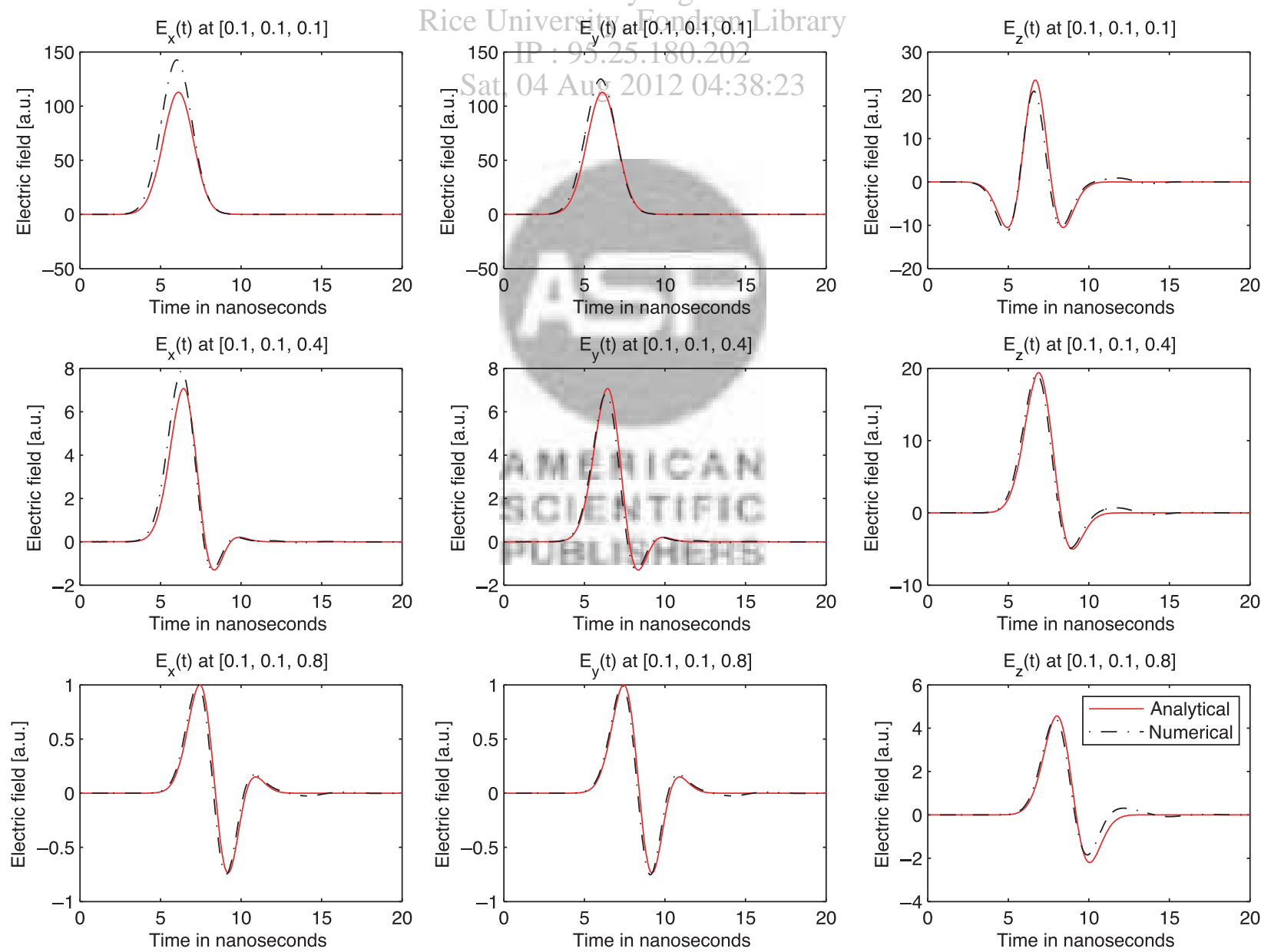

Fig. 5. Plot parameters: mesh size small, i.e. $24^{\prime} 968$ tetrahedra, cf. first column of Table (I) and $2^{\text {nd }}$ order basis functions: we calculate the electric field $\mathbf{E}(\mathbf{x}, t)$ radiated by a Hertzian dipole that is situated at the center of the spherical computational domain with HADES3D and compare the cartesian components of the field vector against the analytical solution in the time domain. Here, we note that using the coarse mesh but $2^{\text {nd }}$ order basis functions we are almost as accurate as in the case of the very fine mesh, Figure (4). Thus, using $2^{\text {nd }}$ order basis functions reduces the computational cost by approximately 2 orders of magnitude. On the other hand, more memory is required when assembling the global finite element matrices. However, memory consumption is a minor issue when compared to the time required for the iterative solution of the linear system of equations, and thus using higher order basis functions is highly efficient. ${ }^{20}$ 


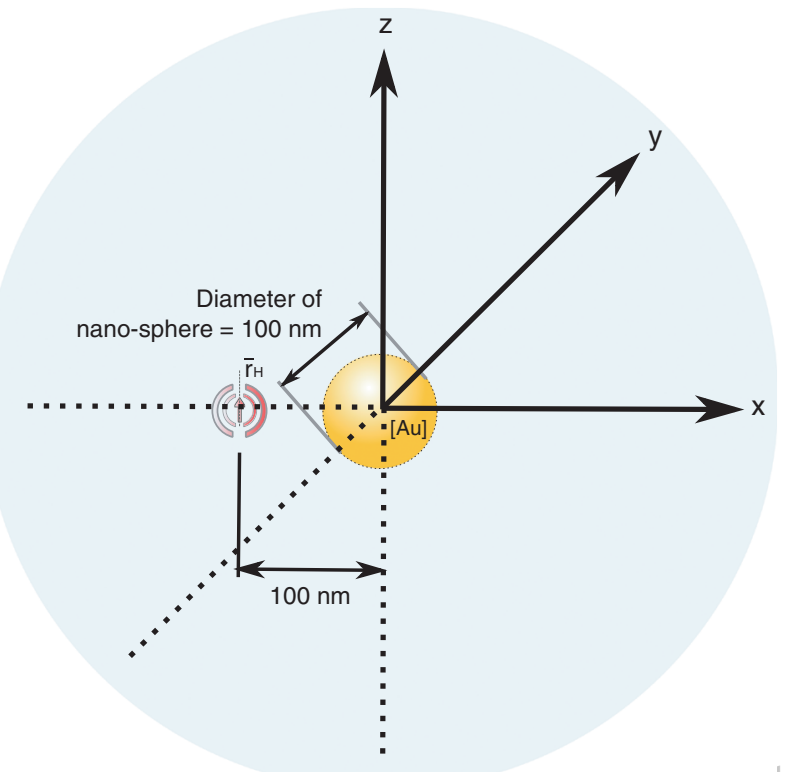

Radius of spherical computational domain $=400 \mathrm{~nm}$

Fig. 6. The Hertzian dipole illuminates a dielectric nano-sphere. We analyze two different cases. The first case models the sphere with a non- 2 dispersive scalar dielectric; the second case models the nano-sphere with a Drude model to capture the dispersive dielectric properties of gold in the optical region of the electromagnetic spectrum.

Hertzian Dipole Benchmark. The objective is to specifically test the capability of the HADES3D code for modeling radiation problems. Thus, we also stress test the performance of the transparent boundary which has been implemented via the $1^{\text {st }}$ order absorbing boundary condition $\left(\mathrm{ABC} 1^{\text {st }}\right)$ to truncate the computational domain. The benchmark concept consists of a single Hertzian dipole, located at the origin of the coordinate system and directed parallel to the $e_{z}$ direction. We adopt a Neumann, i.e., $1^{\text {st }}$ derivative of a Gaussian, pulse for the temporal signature of the radiating, infinitesimally small current density vector, aka. Hertzian dipole. ${ }^{52}$ The shape of the computational domain is a sphere with radius 1 meter which is then tessellated into a tetrahedral mesh. The number of tetrahedral elements of the macro, aka. starting grid, is circa $25^{\prime} 000$ tetrahedra. The complete set of parameters is given in Table I. We calculate the field radiated by the dipole for the macro grid and then for uniformly refined instances of the macro grid. A uniform refinement increases the number of tetrahedra by the factor of 8 . The numerical solution is sampled at 3 different points in space, i.e., in the immediate near field of the dipole, at approximately half the radius of the spherical computational domain and close to the zenith of the computational domain. We have analyzed four different mesh sizes: from circa $25^{\prime} 000$ tetrahedra to 12.8 million tetrahedra, using $1^{\text {st }}$ order basis functions. In Figures $1-5$ we plot the time samples against the analytical solution of the Hertzian dipole. We note that the macro grid with $25^{\prime} 000$ tetrahedra, at best, qualitatively, reproduces the analytical solution of the Hertzian dipole. Solving the electrical field curl-curl equation for increasingly uniformly refined tetrahedral meshs delivers ever better agreement between the numerical and analytical solution, in particular we note Figure 2, then Figures 3 and 4.

Table II. Parameters of the benchmark case where a Hertzian dipole illuminates a dielectric nano-sphere; there are 2 different cases: the first case employs a scalar non-dispersive dielectric permittivitiy; the second case analyzes a gold nano-sphere whose dielectric response is modeled with the Drude model to describe metals in the optical region of the electromagnetic spectrum.

\begin{tabular}{|c|c|c|c|}
\hline Parameter & Non-dispersive $\mathrm{C} / \mathrm{T}$ NT Drude dispersive & Unit & Definition \\
\hline Radius of nano-sphere & PUPA 50 HFME: & $\mathrm{nm}$ & $10^{-9} \mathrm{~m}$ \\
\hline Computational domain shape & Sphere & & \\
\hline Computational domain radius & 400 & $\mathrm{~nm}$ & $10^{-9} \mathrm{~m}$ \\
\hline \multirow[t]{2}{*}{ Background $\epsilon_{r}$} & 1.0 & - & \\
\hline & Dielectric properties of nano-sphere & & \\
\hline$\epsilon_{r}$ & 4.0 & - & \\
\hline$\epsilon_{\infty}$ & 1.0 & - & from [32] \\
\hline Pole frequency $\frac{\omega_{p}}{2 \pi}$ & $2.1963 \cdot 10^{15}$ & $H z$ & from [32] \\
\hline \multirow[t]{2}{*}{ Relaxation frequency $\frac{\gamma_{p}}{2 \pi}$} & $1.7109 \cdot 10^{13}$ & $H z$ & from [32] \\
\hline & Mesh & & \\
\hline Mesh size & $43^{\prime} 854$ & - & \\
\hline Degrees of freedom & $278^{\prime} 894$ & - & \\
\hline \multirow[t]{2}{*}{ Basis function order } & 2 & - & \\
\hline & Time discretization & & \\
\hline Time step $\Delta t$ & 2.0 & as & $10^{-18} \mathrm{~s}$ \\
\hline \multirow[t]{2}{*}{ Number of timesteps } & $10^{\prime} 000$ & - & \\
\hline & Illumination & & \\
\hline Dipole location & $(-100,0,0)$ & $\mathrm{nm}$ & $10^{-9} \mathrm{~m}$ \\
\hline Dipole direction vector & $\mathbf{e}_{z}$ & - & \\
\hline Excitation signal & Sine carrier modulated by Neumann & - & \\
\hline Carrier frequency & $999.31(\lambda=300 \mathrm{~nm})$ & $\mathrm{THz}$ & $10^{12} \mathrm{~Hz}$ \\
\hline Excitation width $\sigma$ & 1.64 & fs & $10^{-15} \mathrm{~s}$ \\
\hline Excitation offset $t_{0}$ & 6.0 & fs & $10^{-15} \mathrm{~s}$ \\
\hline
\end{tabular}


Table III. Parameters for two different optical antenna configurations: (i) this configuration models a single dipole with finite dimensions, consisting of two cylinder shaped rods with a gap between the rods. The rods are terminated at the far end with a hemispherical cap. (ii) this configuration models an array of dipoles, consisting of a driven dipole whose dimensions are equal to case (i), 3 directing dipoles whose lengths obey a logarithmical scaling law and a reflector that is behind the driving dipole, seen from the perspective of the directors. There is similarity to the Yagi-Uda ${ }^{3}$ array antenna with the difference that the directing dipoles become shorter the further away they are from driving dipole. In both cases (i) and (ii) the dipoles are assigned gold material properties which is modeled with the Drude model to describe metals in the optical region of the electromagnetic spectrum.

\begin{tabular}{|c|c|c|c|c|}
\hline Parameter & Single dipole & Dipole array & Unit & Definition \\
\hline Computational domain shape & \multicolumn{2}{|c|}{ Sphere } & & \\
\hline Computational domain radius & \multicolumn{2}{|c|}{1000} & $\mathrm{~nm}$ & $10^{-9} \mathrm{~m}$ \\
\hline Background $\epsilon_{r}$ & \multicolumn{2}{|c|}{1.0} & - & \\
\hline \multicolumn{5}{|c|}{ Drude dielectric properties of dipole metals } \\
\hline$\epsilon_{\infty}$ & & & - & from [32] \\
\hline Pole frequency $\frac{\omega_{p}}{2 \pi}$ & & & $\mathrm{Hz}$ & from [32] \\
\hline Relaxation frequency $\frac{\gamma_{p}}{2 \pi}$ & & & $\mathrm{Hz}$ & from [32] \\
\hline \multicolumn{5}{|c|}{ Mesh, time discretization and excitation parameters } \\
\hline Mesh size & $23^{\prime} 701$ & $140^{\prime} 529$ & - & \\
\hline Basis function order & 2 & 1 & - & \\
\hline Time step $\Delta t$ & 1.0 & 1.0 & as & $10^{-18} \mathrm{~s}$ \\
\hline Number of timesteps & $17^{\prime} 800$ & $20^{\prime} 000$ & - & \\
\hline \multicolumn{5}{|c|}{ Transverse-electric-magnetic (TEM) illumination parameters } \\
\hline TEM reference location & $(-1200,0,0)$ & $(1200,0,0)$ & $\mathrm{nm}$ & $10^{-9} \mathrm{~m}$ \\
\hline Wave direction vector $\mathbf{k}$ & $(1,0,0)$ & $(-1,0,0)$ & $\mathrm{nm}$ & $10^{-9} \mathrm{~m}$ \\
\hline Polarization vector & Rice Un & dren Library & $\mathrm{nm}$ & \\
\hline Excitation signal & Sine c & Neumann & - & \\
\hline Carrier frequency & Sat & m) $38 \cdot 23$ & $\mathrm{THz}$ & $10^{12} \mathrm{~Hz}$ \\
\hline Excitation width $\sigma$ & sals & 04.50 .23 & fs & $10^{-15} \mathrm{~s}$ \\
\hline Excitation offset $t_{0}$ & & & fs & $10^{-15} \mathrm{~s}$ \\
\hline
\end{tabular}

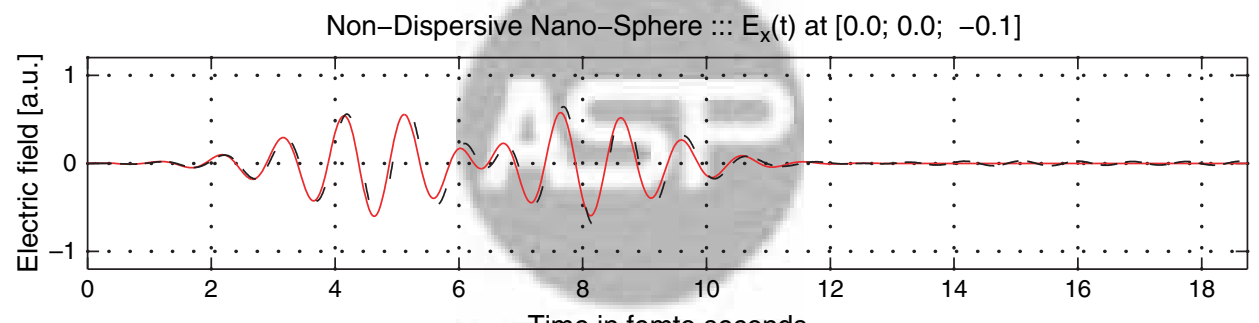

Time in femto seconds

Non-Dispersive Nano-Sphere ::: $\mathrm{E}_{\mathrm{y}}(\mathrm{t})$ at $[0 ; 0.0 ;-0.1]$

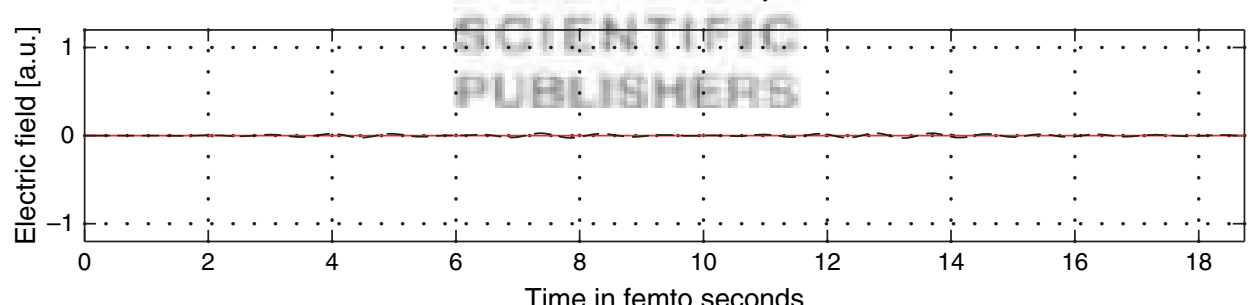

Non-Dispersive Nano-Sphere ::: $\mathrm{E}_{\mathrm{z}}(\mathrm{t})$ at $[0.0 ; 0.0 ;-0.1]$

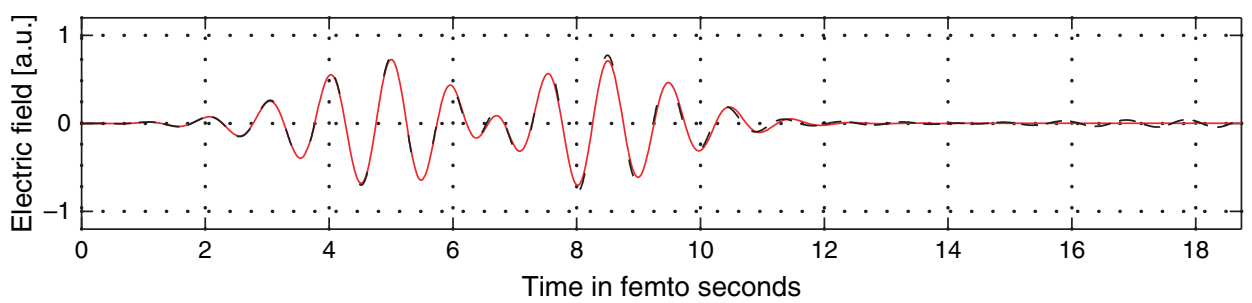

Fig. 7. We calculate the electric field distribution $\mathbf{E}(\mathbf{x}, t)$, radiated by a Hertzian dipole and scattered off a dielectric nano-sphere with the HADES3D code, using $2^{\text {nd }}$ order basis functions. The dipole is parallel to the $\mathbf{e}_{z}$ direction and situated on the $\mathbf{e}_{x}$ axis at $x=-100 \mathrm{~nm}$ whereas the dielectric sphere has radius $r=50 \mathrm{~nm}$ and $\epsilon_{r}=4$. We sample the electric field at the location $\{0,0,-100\} \mathrm{nm}$ and compare it component by component to the analytical solution ${ }^{26}$ converted from the frequency domain into the time domain. The analytical solution is the red line and the numerical solution is the dashed line in black. 
Remarkably, when comparing the largest tetrahedral mesh using $1^{\text {st }}$ order basis functions, Figure 4 , with the solution for the coarse tetrahedral mesh using $2^{\text {nd }}$ order basis functions, Figure 5, we note that employing the coarse mesh with circa $25^{\prime} 000$ tetrahedra but employing higher order basis functions delivers almost as good a solution as the most refined version of the mesh with ca. 12.8 million tetrahedra but using $1^{\text {st }}$ order basis functions only. Hence, by using higher order basis functions we have reduced the necessary computational effort by between 2 and 3 orders of magnitude. Thus, using higher order basis functions is considerably more efficient, in order to increase accuracy, when compared to uniform mesh refinement. ${ }^{20} \mathrm{We}$ also comment that the numerical analysis of the Hertzian dipole and comparison of the electric field sampled in the immediate near field represents a challenging test for almost any computational electromagnetics code since the dipole field varies with $r^{-3}$ in the immediate near field.

Dipole in Front of a Dielectric Nano-Sphere. This benchmark tests the capability of the HADES3D code to model a complicated electromagnetic near-field problem. It employs a Hertzian dipole that illuminates a dielectric nano-sphere; the dipole is situated in close proximitiy to it. In particular, we calculate the electric field distribution
$\mathbf{E}(\mathbf{x}, t)$, radiated by a Hertzian dipole and scattered off a dielectric nano-sphere with the HADES3D code using $2^{\text {nd }}$ order basis functions. The dipole is parallel to the $\mathbf{e}_{z}$ direction and situated on the $\mathbf{e}_{x}$ axis at $x=-100 \mathrm{~nm}$ whereas the dielectric sphere has radius $r=50 \mathrm{~nm}$ and is situated at the origin, cf. Figure 6 for the geometry. The shape of the computational domain is a sphere with radius $r=400 \mathrm{~nm}$. The nano-sphere is analyzed for two different sets of dielectric permittivity: the first set assumes the nano-sphere being made of a non-dispersive dielectric material with $\epsilon_{r}=4.0$ while the second case models the nano-sphere using the Drude dispersive dielectric model for gold. ${ }^{32}$ We have chosen this electromagnetic problem since it can be solved analytically in the frequency domain ${ }^{26}$ and then converted into the time domain via the fast Fourier transform (FFT). Thus, we can directly compare the numerical solution delivered by the HADES3D code to the analytical solution. The complete set of parameters is given in Table II. We sample the numerical solution at 3 different locations and plot the cartesian components of the numerical solution against the analytical solution:

(1) sampling location $\{0,0,-100\} \mathrm{nm}$ : Figures 7 and 10 for the non-dispersive and the Drude dispersive case, respectively;

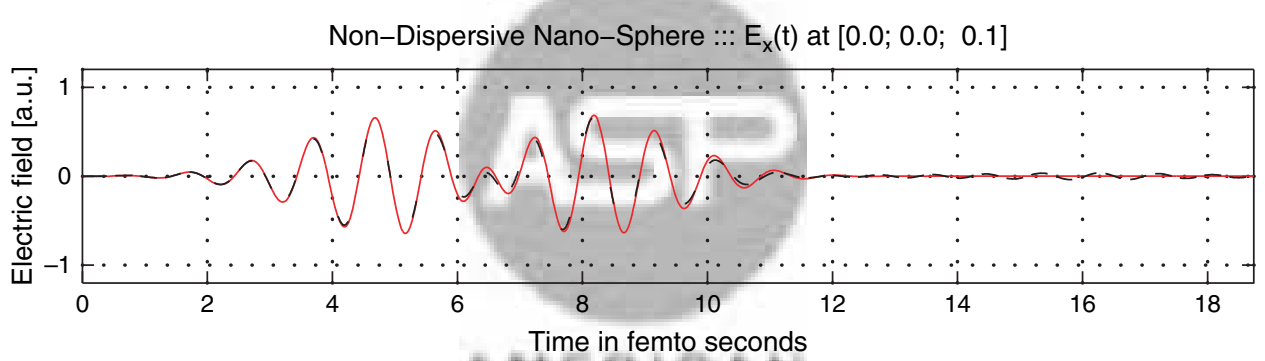

Non-Dispersive Nano-Sphere :.: $\mathrm{E}_{\mathrm{y}}(\mathrm{t})$ at $[0.0 ; 0.0 ; 0.1]$
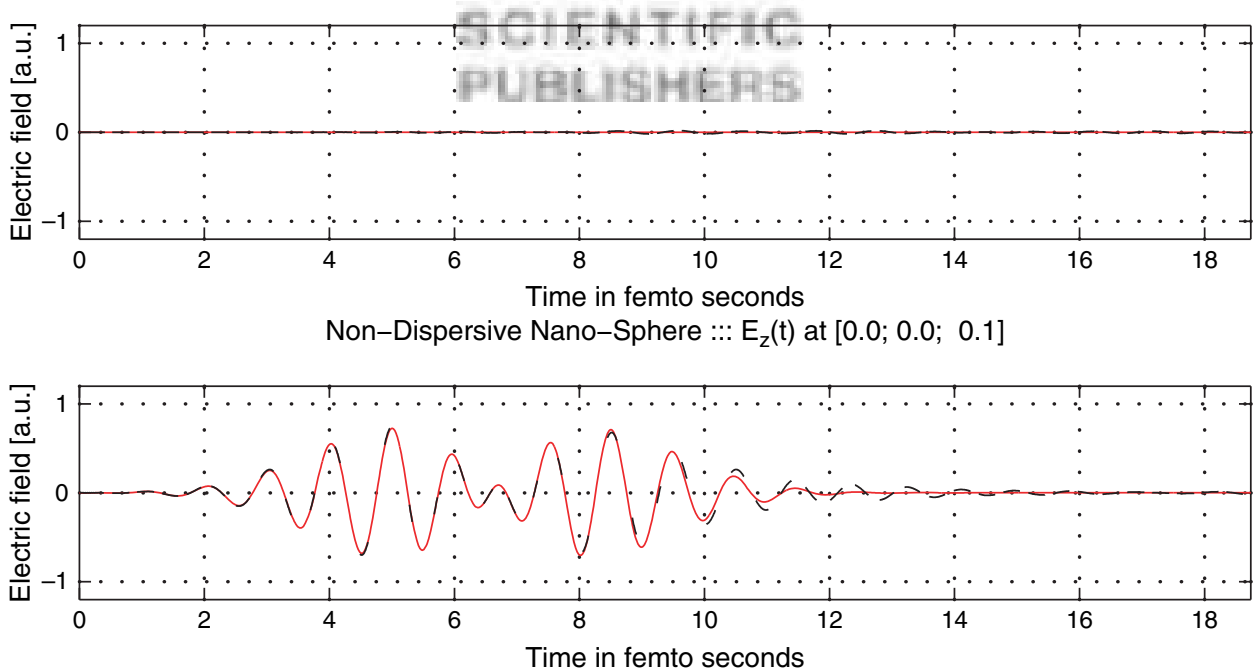

Fig. 8. We calculate the electric field distribution $\mathbf{E}(\mathbf{x}, t)$, radiated by a Hertzian dipole and scattered off a dielectric nano-sphere with the HADEs $3 \mathrm{D}$ code, using $2^{\text {nd }}$ order basis functions. The dipole is parallel to the $\mathbf{e}_{z}$ direction and situated on the negative $\mathbf{e}_{x}$ axis at $x=-100 \mathrm{~nm}$ whereas the dielectric sphere has radius $r=50 \mathrm{~nm}$ and $\epsilon_{r}=4$. We sample the electric field at the location $\{0,0,100\} \mathrm{nm}$ and compare it component by component to the analytical solution, ${ }^{26}$ converted from the frequency domain into the time domain. The analytical solution is the red line and the numerical solution is the dashed line in black. 


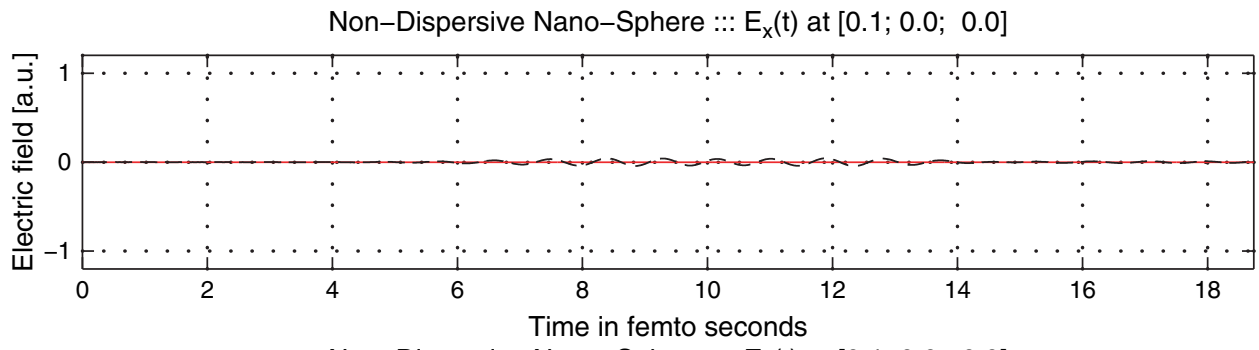

Non-Dispersive Nano-Sphere ::: $\mathrm{E}_{\mathrm{y}}(\mathrm{t})$ at $[0.1 ; 0.0 ; 0.0]$

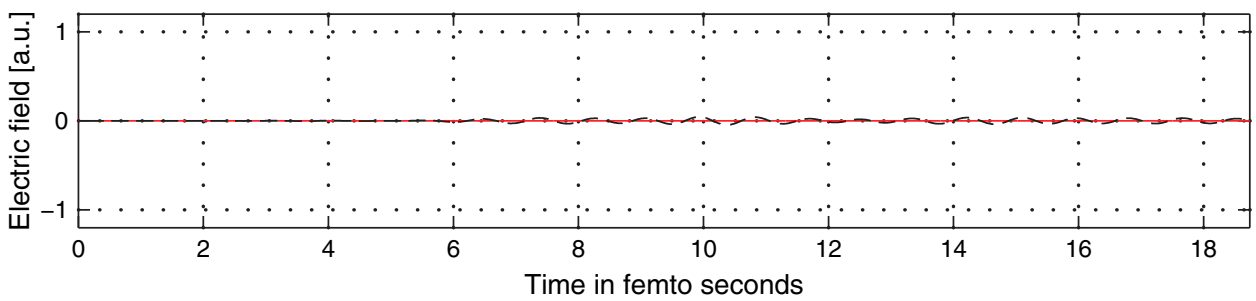

Non-Dispersive Nano-Sphere ::: $\mathrm{E}_{\mathrm{z}}(\mathrm{t})$ at $[0.1 ; 0.0 ; 0.0]$

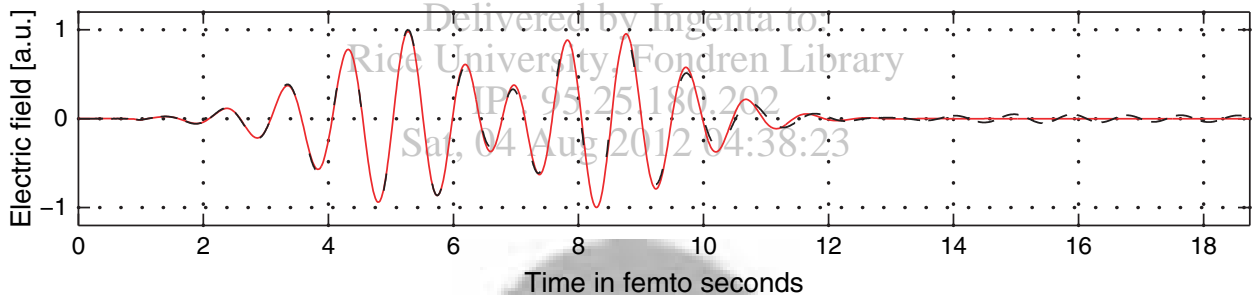

Fig. 9. We calculate the electric field distribution $\mathbf{E}(\mathbf{x}, t)$, radiated by a Hertzian dipole and scattered off a dielectric nano-sphere with the HADES3D code, using $2^{\text {nd }}$ order basis functions. The dipole is parallel to the $\mathbf{e}_{z}$ direction and situated on the negative $\mathbf{e}_{x}$ axis at $x=-100 \mathrm{~nm}$ whereas the dielectric sphere has radius $r=50 \mathrm{~nm}$ and $\epsilon_{r}=4$. We sample the electric field at the location $\{100,0,0\} \mathrm{nm}$ and compare it component by component to the analytical solution, ${ }^{26}$ converted from the frequency domain into the time domain. The analytical solution is the red line and the numerical solution is the dashed line in black.

(2) sampling location $\{0,0,100\} \mathrm{nm}$ : Figures 8 and 11 for the non-dispersive and the Drude dispersive case, respectively; and

(3) sampling location $\{100,0,0\} \mathrm{nm}$ : Figures $\overline{9}$ and $\overline{12}$ for the non-dispersive and the Drude dispersive case, respectively.

We comment that we have good qualitative and quantitative agreement between the numerical (dashed line) and the analytical (red line) solution, given the fact that this benchmark problem represents a challenging configuration for almost any computational electromagnetics code. This is particularly true since the dipole's radiated field varies with $r^{-3}$ in its immediate vicinity and, thus, presents formidable requirements onto the finite element method to model a field that rapidly decays over a relatively short distance. We note that in the dispersive case there is a certain amount of ringing that only decays slowly. We believe, backed by the convergence observed in the case of the Hertzian dipole radiating into vacuum, that using an even more refined mesh will reduce ringing. Overall, we state that we can anaylze electromagnetic near-field problems both for non-dispersive and dispersive material properties with confidence.
Number of Iterations. Remarkably, the number of iterations required for the iterative solver to converge is relatively stable. In the case of $1^{\text {st }}$ order basis functions approximately 70-90 iterations are required while in the case of $2^{\text {nd }}$ order basis functions approximately 200-230 iterations are required. It is understood that the quality of the mesh immensely influences the number of iterations. A tetrahedral mesh of poor quality will certainly destroy convergence, easily requiring hundreds of iterations until the state of convergence can be reached, if at all.

\section{RESULTS AND DISCUSSION}

We show the visualization of the electric field distribution for the

(1) single dipole optical antenna in Figures 14-15 and the

(2) dipole array antenna in Figure 16 respectively.

In particular, we plot the magnitude of the electric field vector. We present both close-up and total views.

Single Dipole Antenna. Here we observe a standing wave pattern in the longitudinal direction of the antenna, Figures 14(a) and 15(a). Also, there is electric field enhancement between the gaps of the single dipole arms. 

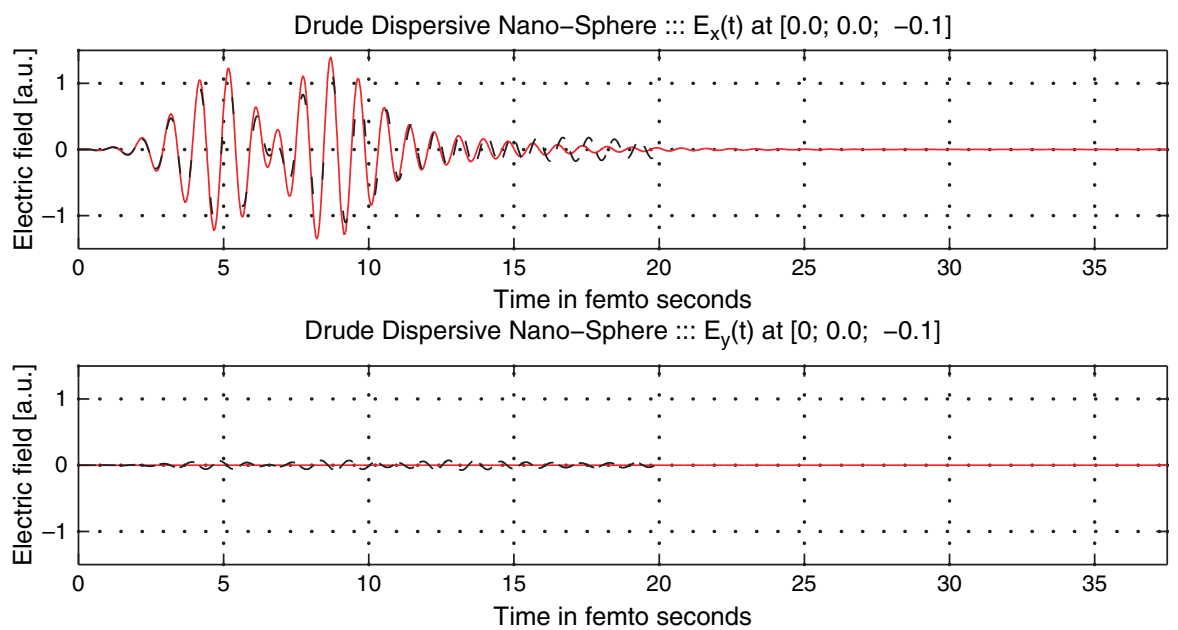

Drude Dispersive Nano-Sphere ::: $\mathrm{E}_{\mathrm{z}}(\mathrm{t})$ at $[0.0 ; 0.0 ;-0.1]$

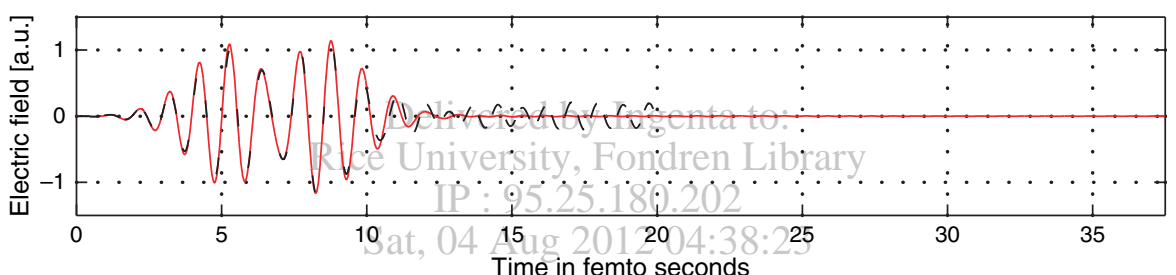

Fig. 10. We calculate the electric field distribution $\mathbf{E}(\mathbf{x}, t)$, radiated by a Hertzian dipole and scattered off a gold nano-sphere with the HADEs3D code, using $2^{\text {nd }}$ order basis functions. The dipole is parallel to the $\mathbf{e}_{z}$ direction and situated on the negative $\mathbf{e}_{x}$ axis at $x=-100 \mathrm{~nm}$ whereas the dielectric sphere has radius $r=50 \mathrm{~nm}$. We sample the electric field at the location $\{0,0,-100\} \mathrm{nm}$ and compare it component by component to the analytical solution, ${ }^{26}$ converted from the frequency domain into the time domain. The analytical solution is the red line and the numerical solution is the dashed line in black.

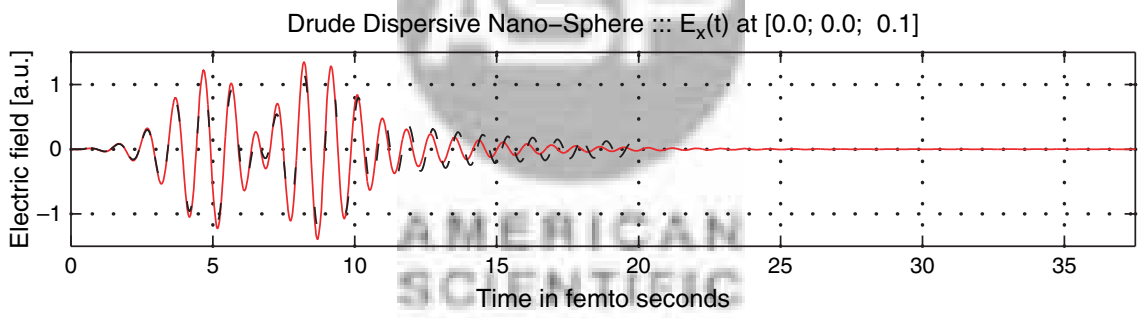

Drude Dispersive Nano-Sphere :.: $\mathrm{E}_{\mathrm{y}}(\mathrm{t})$ at $[0.0 ; 0.0 ; 0.1]$

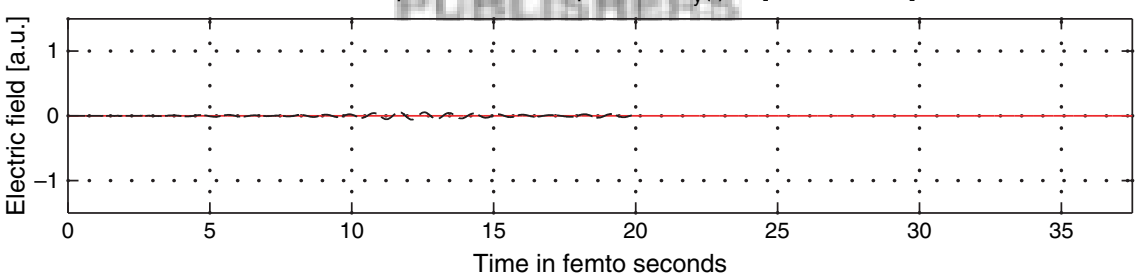

Drude Dispersive Nano-Sphere ::: $\mathrm{E}_{\mathrm{z}}(\mathrm{t})$ at $[0.0 ; 0.0 ; 0.1]$

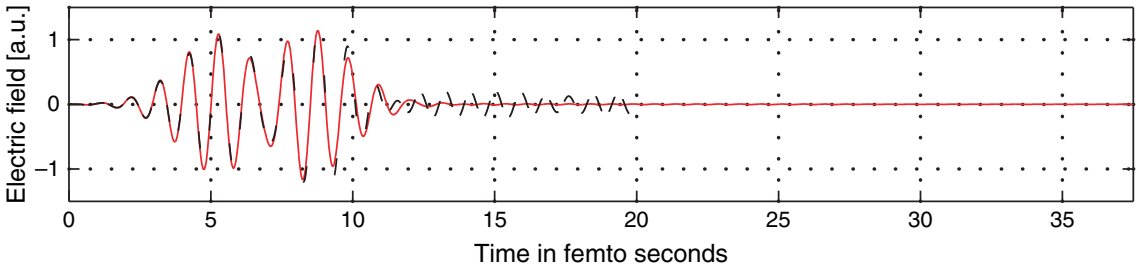

Fig. 11. We calculate the electric field distribution $\mathbf{E}(\mathbf{x}, t)$, radiated by a Hertzian dipole and scattered off a gold nano-sphere with the HADES3D code, using $2^{\text {nd }}$ order basis functions. The dipole is parallel to the $\mathbf{e}_{z}$ direction and situated on the negative $\mathbf{e}_{x}$ axis at $x=-100 \mathrm{~nm}$ whereas the dielectric sphere has radius $r=50 \mathrm{~nm}$. We sample the electric field at the location $\{0,0,100\} \mathrm{nm}$ and compare it component by component to the analytical solution, ${ }^{26}$ converted from the frequency domain into the time domain. The analytical solution is the red line and the numerical solution is the dashed line in black. 
Drude Dispersive Nano-Sphere ::: $\mathrm{E}_{\mathrm{x}}(\mathrm{t})$ at $[0.1 ; 0.0 ; 0.0]$

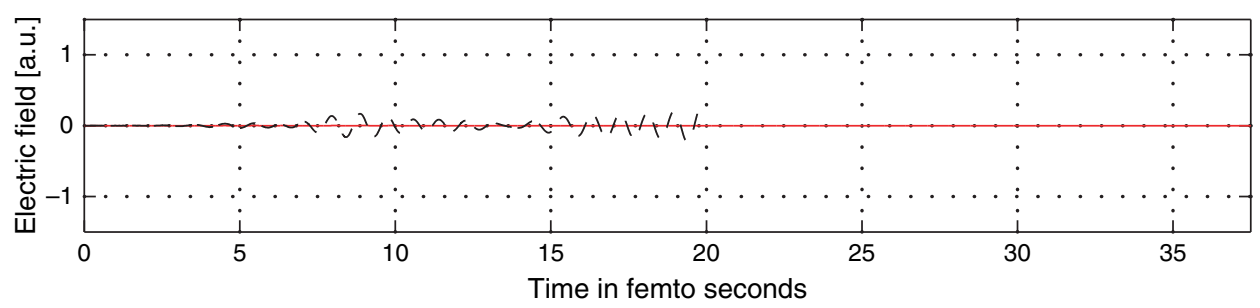

Drude Dispersive Nano-Sphere :.: $\mathrm{E}_{\mathrm{y}}(\mathrm{t})$ at $[0.1 ; 0.0 ; 0.0]$

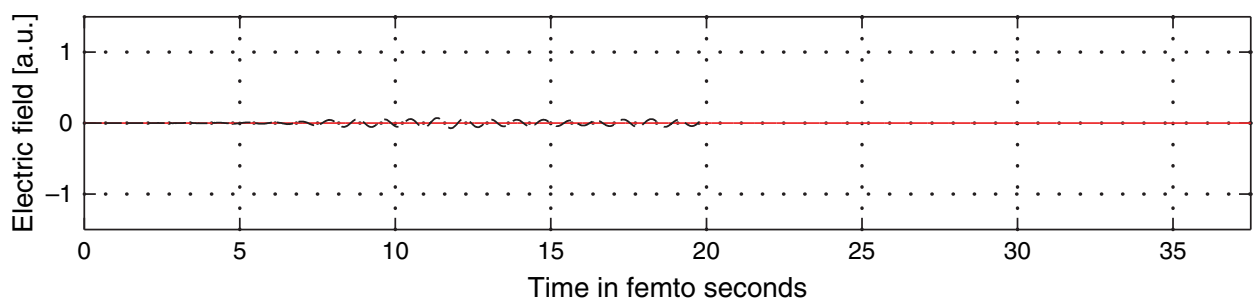

Drude Dispersive Nano-Sphere :.: $\mathrm{E}_{\mathrm{z}}(\mathrm{t})$ at $[0.1 ; 0.0 ; 0.0]$

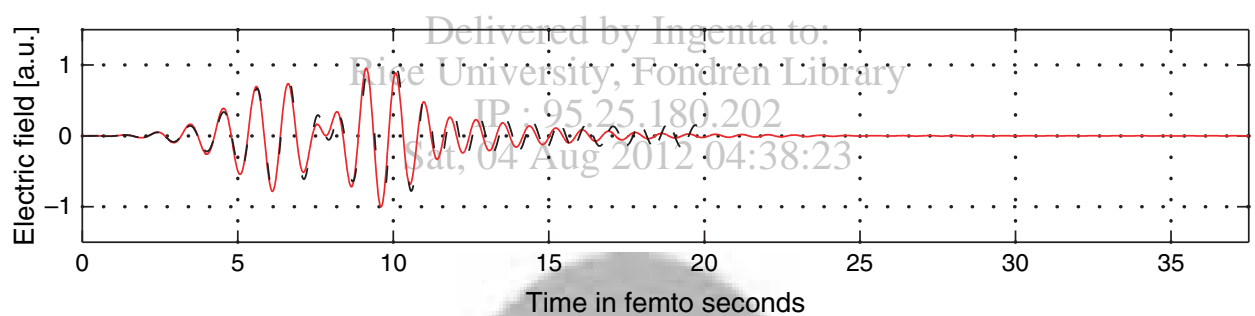

Fig. 12. We calculate the electric field distribution $\mathbf{E}(\mathbf{x}, t)$, radiated by a Hertzian dipole and scattered off a gold nano-sphere with the HADEs $3 \mathrm{D}$ code, using $2^{\text {nd }}$ order basis functions. The dipole is parallel to the $\mathbf{e}_{z}$ direction and situated on the negative $\mathbf{e}_{x}$ axis at $x=-100 \mathrm{~nm}$ whereas the dielectric sphere has radius $r=50 \mathrm{~nm}$. We sample the electric field at the location $\{100,0,0\} \mathrm{nm}$, i.e. at the mirror location of the dipole source, and compare it component by component to the analytical solution, ${ }^{26}$ converted from the frequency domain into the time domain. The analytical solution is the red line and the numerical solution is the dashed line in black.

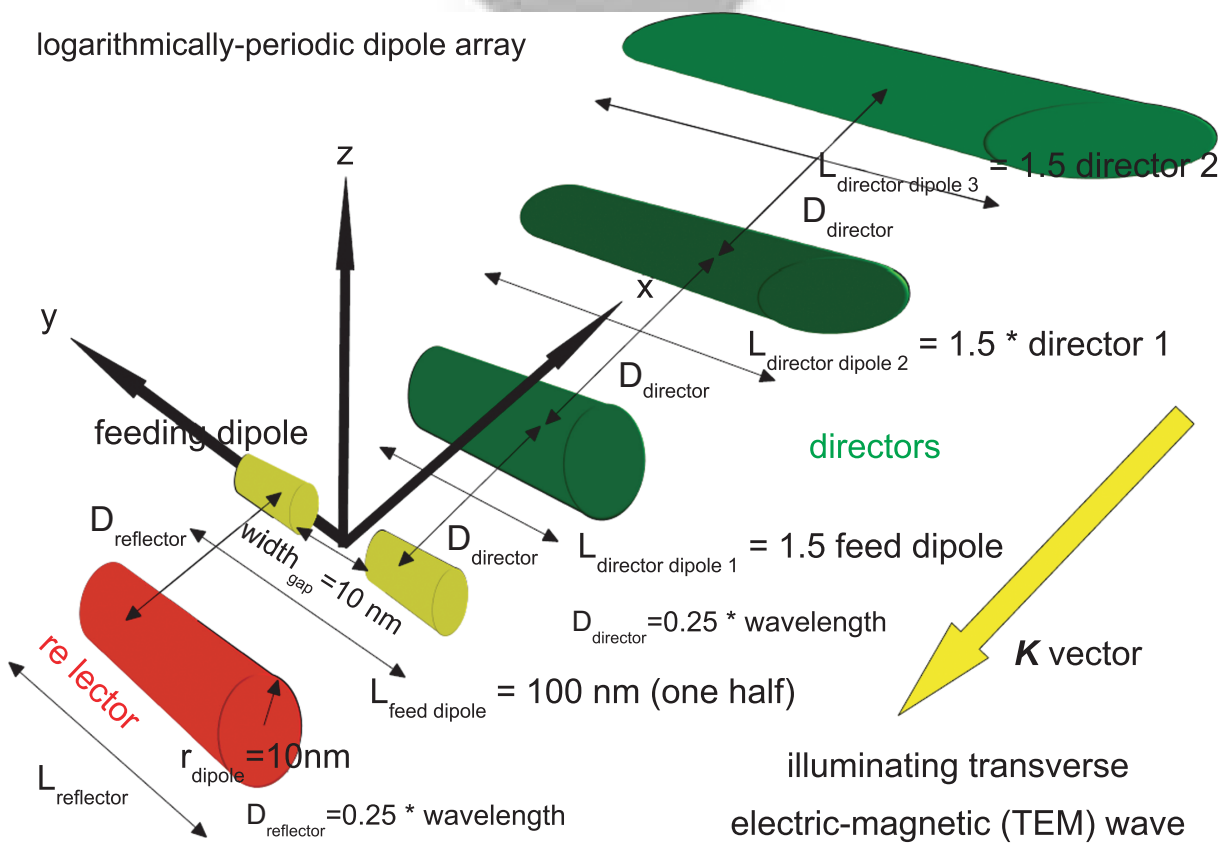

Fig. 13. We calculate the total electric field for an array of logarithmically-periodic dipoles in the optical region. The array antenna is illuminated with a transverse-electric-magnetic (TEM) wave incoming from the positive $x$-axis with the wave direction vector $\mathbf{k}=(-1,0,0)$. The computational domain has spherical shape with radius $r_{\text {sphere }}=1000 \mathrm{~nm}$. We have terminated the mesh with a $1^{\text {st }}$ order absorbing boundary condition. For the complete set of simulation parameters cf. Table (III). 
(a)

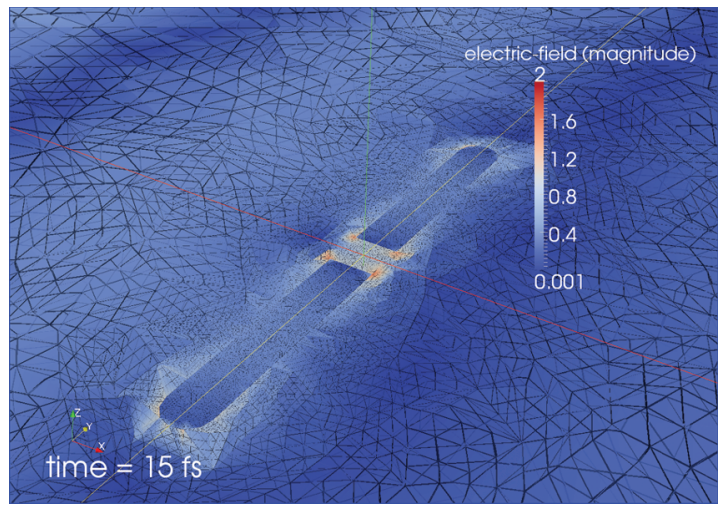

(b)

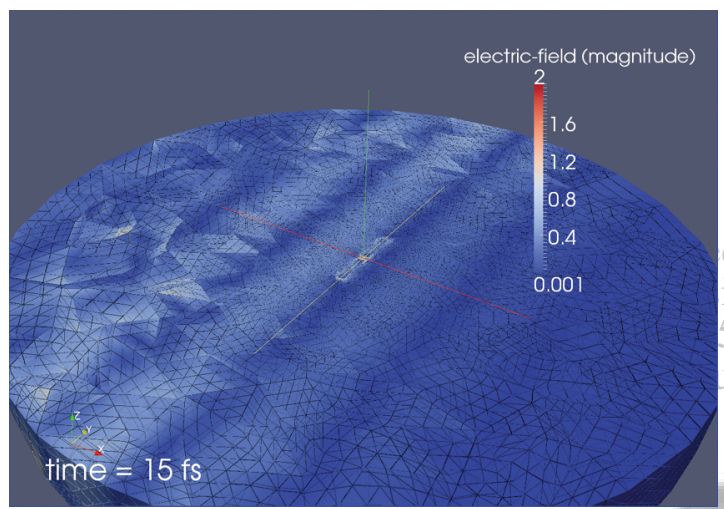

Fig. 14. We plot the magnitude of the electric field distribution for the simple dipole antenna, consisting of 2 single arms, separated by a short gap with width $=10 \mathrm{~nm}$. The electromagnetic wave illuminates the dipole antenna from the negative $x$ axis with $\mathbf{e}_{y}$ polarization.

While the TEM wave propagates over and through the structure we clearly see the resonant nature of the electric field on the antenna. This observation is remarkable since in many other studies frequency domain methods are employed. Here, we have demonstrated resonant behavior in the time domain. While the tetrahedral mesh is relatively coarse, this only applies to regions that are further away from the finite sized dipole antenna. Here, we profit from the capability of the FETD method to resolve complicated, curved geometry through modeling with level of detail (LoD); we have prepared a mesh that is fine in the immediate vicinity of the dipole and becomes coarser further away from the dipole. We mention that this analysis uses $2^{\text {nd }}$ order basis functions. The visualization of the fields reveals sub-wavelength features for metallic structures in the optical region of the frequency spectrum. We have also analyzed this problem with $1^{\text {st }}$ order basis functions and found analogous standing wave patterns. We comment that the single dipole could be made even shorter if only a resonance at $\lambda=532 \mathrm{~nm}$ is desired..$^{8,31}$

Dipole Array Antenna. Here, we have used $1^{\text {st }}$ order basis functions. We also observe a standing wave pattern on all the dipole's elements when the TEM wave propagates over and through the antenna's finite sized rods, Figure 16(a). Since there is more than one dipole we also (a)

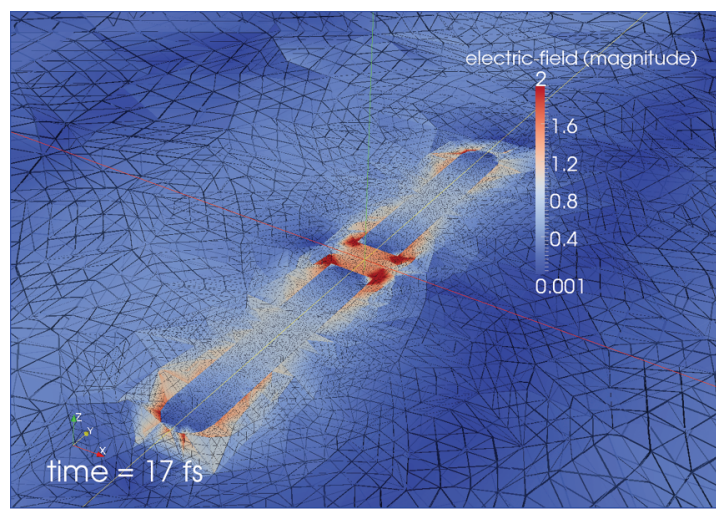

(b)

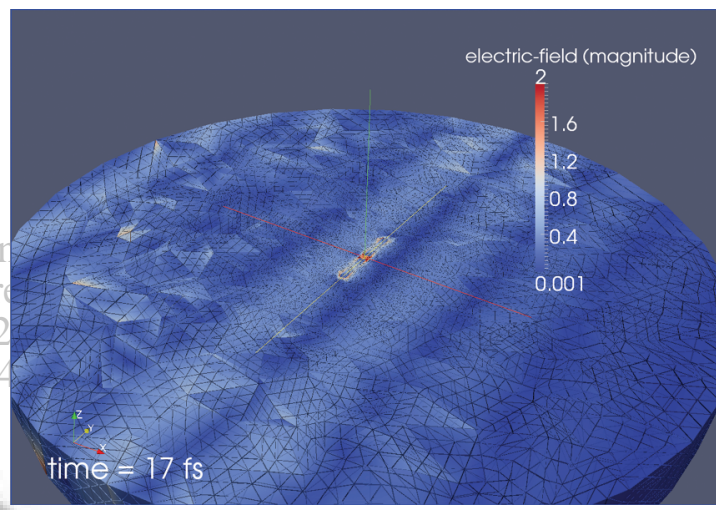

Fig. 15. We plot the magnitude of the electric field distribution for the simple dipole antenna, consisting of 2 single arms, separated by a short gap with width $=10 \mathrm{~nm}$. The electromagnetic wave illuminates the dipole antenna from the negative $x$ axis with $\mathbf{e}_{y}$ polarization.

observe the different phases of the resonant fields on the driving, director and reflector dipoles. The mesh employed for this analysis counts approximately $140^{\prime} 000$ tetrahedra while the mesh used in the analysis of the single dipole only counts some $23^{\prime} 000$ tetrahedra. This is caused by the fact that the array antenna employs more than one delicately shaped dipole rod. Thus, the number of regions with fine features in the mesh increases. Still, the level of detail employed in the mesh generation process allows to tessellate the original geometry in such a way that the efficiency of spatial discretization is maintained. Similar as in the case of the single dipole antenna we also observe field enhancement in the gap region of the driving dipole.

Synthesis. We have analyzed complicated optical antenna systems in fully 3-dimensional space and demonstrated the existence of resonant field behavior in both cases. We comment that using an even finer mesh will certainly benefit the degree of reality but on the other hand must be balanced with calculation times that are here on the order of hours. The careful selection of the basis function order and the generation of the detailed tetrahedral mesh allows us to increase modeling accuracy while simultaneously keeping computational cost affordable. Even with these medium sized meshes we can observe the principal characteristics and relevant features of optical 
(a)

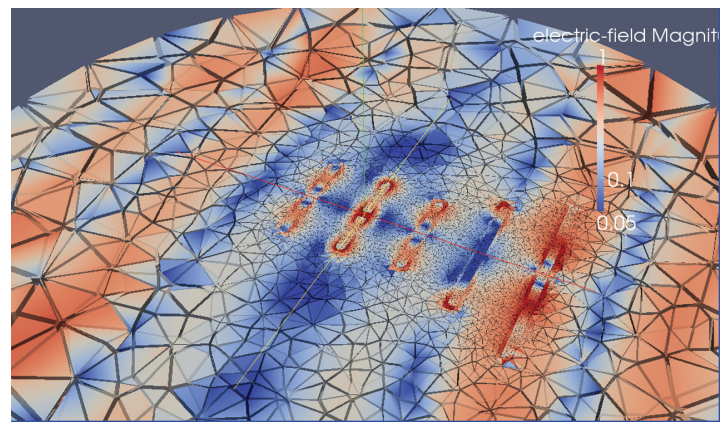

(b)

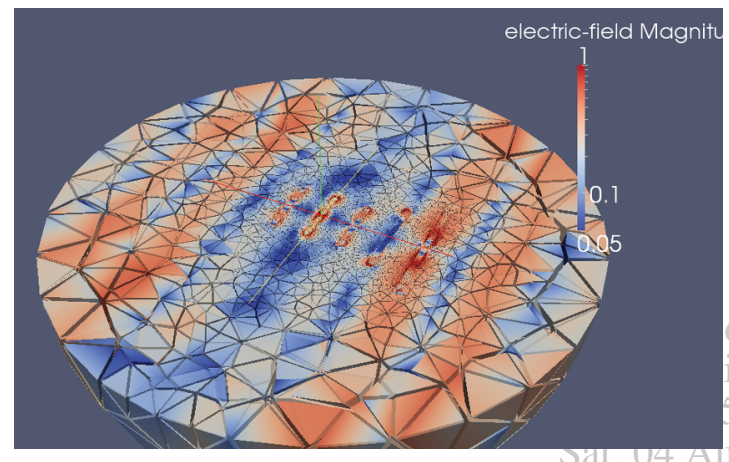

Fig. 16. We plot the magnitude of the electric field distribution for the dipole array antenna, consisting of a 5 different dipoles, i.e. a driver dipole that consists of 2 single arms separated by a gap with width $=$ $10 \mathrm{~nm}$, a reflector dipole situated on the negative $\mathbf{e}_{x}$ axis and 3 director dipoles on the positive $\mathbf{e}_{x}$ axis. The TEM wave illuminates the dipole antenna from the positive $x$ axis with $\mathbf{e}_{y}$ polarization. sphere illuminated by a Hertzian dipole. Using an even finer mesh for the discretization of the nano-sphere such ringing will decrease but simultaneously increase computational cost. The HADES3D code has been optimized to profit from today's standard distributed-memory parallel computers in order to accelerate the time-domain integration which requires a large linear system be solved at every time step. We have then analyzed optical antennas that employ finite sized dipole elements. There, we have numerically calculated the electric field distribution for a single dipole and a dipole array antenna with logarithmically-periodic scaled dipole elements. Resonant field behavior, both on the single dipole and the dipole array, has been demonstrated. The existence of resonances is tightly coupled to the employment of dispersive dielectric properties for modeling metals in the optical region. In particular, we have demonstrated sub-wavelengh characteristics of the field, especially in the gap region of the driving dipole. The optical dipole antennas have been modeled with finite sized geometry.

Conclusions. The HADES3D code can therefore be used to analyze nano-optical problems in 3 dimensions and the time domain with confidence. In order to further increase the accuracy of the code for modeling dispersive dielectric materials, the convolution operation will probably profit when using a higher order interpolation scheme for the electric field evaluation. At present, linear interpolation is used. Nevertheless, the HADES3D code is, to a degree, in a unique position in that there are not many other time domain computational electrodynamics codes with full 3dimensional capability, that can solve large nano-optical problems with efficiency, by using level of detail in the spatial discretization process, and still deliver those results I within reasonable time. This is particularly so because the solution process has been parallelized. We also emphasize that the quality of the tetrahedral mesh is paramount in the iterative solution of the linear system of equations. Mesh quality and solver speed are intricately linked.

Further Work. We will need to implement a nearto-far-field transformation based on a Green's functions approach $^{46}$ so that we can more easily compare numerical to experimental results. Also, the optical antennas are now investigated via incoming plane wave time domain sources. In order to study the antennas in the radiation case we need to think on proper excitation mechanisms that cause the antenna to radiate. For example, one could devise a voltage source located between the arms of the driver dipole in order to excite currents flowing along the dipole arms. While the single dipole antenna was analyzed with $2^{\text {nd }}$ order basis functions, the dipole array antenna was studied with $1^{\text {st }}$ order basis functions. We comment that using $2^{\text {nd }}$ order basis functions will increase the number of DoF by an approximate factor of 6.5 , thus also increasing the computational work load. On the other hand, it will increase modeling accuracy considerably albeit at the cost 
of longer computation times. Therefore, the study of more efficient preconditioning schemes, which exploit the hierarchical nature of the linear system matrix, will prove fruitful. This is particularly true when even higher, $3^{\text {rd }}$ order basis functions are employed. From the perspective of field emitter modeling, we need to include the pyramidally shaped tip in order to model the interaction between the antenna structure and the emitter where field enhancement is primarily desired. This will further complicate the analysis but profit immensely from the level of detail enabled by the finite element time domain method in electromagnetics. Also, even more realistic dispersive material modeling will require the addition of the Lorentz model which we are currently validating.

Acknowledgments: This work was supported by a grant from the Swiss National Supercomputing Centre (CSCS) under project ID Beam Dynamics for the PSI$X F E L$ and the High Power Cyclotron Upgrade and project ID Nano-Optics for Advanced Photo-Cathodes. Benedikt Oswald acknowledges with gratitude the support provided by Tim Stitt at CSCS in order to obtain permission for running some jobs in the high impact queue of the Monte Rosa machine. Arya Fallahi acknowledges the support from the Swiss National Science Foundation (SNSF) under Contract No. 200021-126843/1.

\section{References}

1. R. Bakker, A. Boltasseva, Z. Liu, R. Pedersen, S. Gresillon, A. Kildishev, V. Drachev, and V. Shalaev, Opt. Express 15, 13682 (2007).

2. R. M. Bakker, V. P. Drachev, Z. Liu, H.-K. Yuan, R. H. Pedersen, A. Boltasseva, J. Chen, J. Irudayaraj, A. V. Kildishev, and V. M. Shalaev, New J. Phys. 10, 125022 (2008).

3. C. A. Balanis, Antenna Theory, 2nd edn., John Wiley \& Sons, Inc. New York (1997)

4. P. Bastian, Parallele adaptive Mehrgitterverfahren, Skripten zur Numerik, Teubner, Stuttgart, Germany (1996).

5. P. Bastian, M. Blatt, A. Dedner, C. Engwer, R. Kloefkorn, M. Ohlberger, and O. Sander, The DUNE-PROJECT, aka, the Distributed and Unified Numerics Environment, www.dune-project.org.

6. P. Bastian, M. Blatt, A. Dedner, C. Engwer, R. Klöfkorn R. Kornhuber, M. Ohlberger, and O. Sander, Computing 82, 121 (2008).

7. P. Bastian, M. Blatt, A. Dedner, C. Engwer, R. Klöfkorn, M. Ohlberger, and O. Sander, Computing 82, 103 (2008).

8. P. Bharadwaj, B. Deutsch, and L. Novotny. Advances in Optics and Photonics 1, 438 (2009).

9. P. Bharadwaj and L. Novotny, Opt. Express 15, 14266 (2007).

10. M. Born and E. Wolf, Principles of Optics, 7th edn., Cambridge University Press, Cambridge (1999).

11. C. A. Brau, Nucl. Instrum. Meth. A 393, 426 (1997).

12. J. Feldhaus, J. Arthur, and J. B. Hastings, J. Phys. B-At Mol Opt 38, S799 (2005).

13. R. H. Fowler and L. Nordheim. Proceedings of the Royal Society of London. Series A 119, 173 (1928).

14. R. Ganter, R. Bakker, C. Gough, S. C. Leemann, M. Paraliev, M. Pedrozzi, F. Le Pimpec, V. Schlott, L. Rivkin, and A. Wrulich, Phys. Rev. Lett. 100, 064801 (2008).
15. G. H. Golub and C. F. van Loan, Matrix Computations, 2nd edn., John Hopkins, Baltimore, Maryland (1990).

16. W. Gropp, E. Lusk, and A. Skjellum. USING MPI-Portable Parallel Programming with the Message-Passing Interface, 2nd edn., Scientific and Engineering Computation Series, The MIT Press, The MIT Press, Cambridge, Massachusetts, London, England (1995).

17. B. Hecht, B. Sick, U. P. Wild, V. Deckert, R. Zenobi, and O. J. F. Martin, J. Chem. Phys. 112, 7761 (2000).

18. H. F. Hofmann, T. Kosako, and Y. Kadoya, New J. Phys. 9, 217 (2007).

19. P. Hommelhoff, Y. Sortais, A. Aghajani-Talesh, and M. A. Kasevich, Phys. Rev. Lett. 96, 077401 (2006).

20. J. Jin, The Finite Element Method in Electromagnetics, 2nd edn., Wiley-Interscience, New York (2002).

21. R. Kappeler, D. Erni, C. Xudong, and L. Novotny, J. Comput. Theor. Nanos. 4, 686 (2007)

22. W. E. King, G. H. Campbell, A. Frank, B. Reed, J. F. Schmerge, B. J. Siwick, B. C. Stuart, and P. M. Weber, J. Appl. Phys. 97, 111101 (2005).

23. E. Kirk, S. Tsujino, and T. Vogel, J. Vac. Sci. Technol. B 27, 1813 (2009).

24. M. W. Knight and N. J. Halas, New J. Phys. 10, 105006 (2008).

25. A. Lewis, H. Taha, A. Strinkovski, A. Manevitch, A. Khatchatouriants, R. Dekhter, and E. Ammann, Nat. Biotechnol. 21, 1378 (2003).

26. L.W. Li, P. S. Kooi, M. S. Leong, and T. S. Yee, IEEE T. Microw. Theory, 42, 2302 (1994).

27. A. Mohammadi, V. Sandoghdar, and M. Agio, New J. Phys. 10, 105015 (2008).

28. A. Mohammadi, F. Kaminski, V. Sandoghdar, and M. Agio, Int. J. Nanotechnol. 6, 902 (2009).

29. A. Mohammadi, V. Sandoghdar, and M. Agio, J. Comput. Theor. Nanosci. 6, 2024 (2009).

30. P. Muehlschlegel, H. J. Eisler, O. Martin, B. Hecht, and D. Pohl, Science 308, 1607 (2005).

31. L. Novotny, Phys. Rev. Lett. 98, 266802 (2007).

32. L. Novotny and B. Hecht, Principles of Nano-Optics, Cambridge University Press (2006)

33. M. Ohtsu and K. Kobayashi, Optical Near Fields, 1st edn., Advanced Texts in Physics, Springer, Berlin-Heidelberg (2004).

34. B. Oswald and P. Leidenberger, J. Comput. Theor. Nanosci. 6, 784 (2009).

35. B. Oswald, P. Leidenberger, and C. Hafner, J. Comput. Theor. Nanosci. 5, 735 (2008)

36. B. Oswald, S. Tsujino, and P. Leidenberger, Modeling nanometer structured laser induced field emission, In Shin ichi Kurokawa and KEK Katsunobu Oide, editors, IPAC'10-Proceedings, Kyoto, Japan (2010).

37. D. W. Pohl, W. Denk, and M. Lanz, Appl. Phys. Lett. 44, 651 (1984).

38. A. D. Rakic, A. B. Djurišic, J. M. Elazar, and M. L. Majewski, $\underline{A p p l}$. Opt. 37, 5271 (1998).

39. L. Rogobete, F. Kaminski, M. Agio, and V. Sandoghdar, Opt. Lett. 32, 1623 (2007).

40. C. Ropers, C. C. Neacsu, T. Elsaesser, M. Albrecht, M. B. Raschke, and C. Lienau, Nano Lett. 7, 2784 (2007).

41. C. Ropers, D. R. Solli, C. P. Schulz, C. Lienau, and T. Elsaesser, Phys. Rev. Lett. 98, 043907 (2007).

42. C. Ropers, C. C. Neacsu, M. B. Raschke, M. Albrecht, C. Lienau, and T. Elsaesser, Jpn. J. Appl. Phys. 47, 6061 (2008).

43. Y. Saad, Iterative Methods for Sparse Linear Systems, 2nd edn., Society for Industrial and Applied Mathematics, 3600 University City Center, Philadelphia, PA (2003).

44. C. A. Spindt, J. Appl. Phys. 39, 3504 (1968).

45. C. A. Spindt, I. Brodie, L. Humphrey, and E. R. Westerberg, J. Appl. Phys. 47, 5248 (1976). 
46. A. Taflove and S. C. Hagness, Computational Electrodynamics: The Finite Difference Time Domain Method, 3rd edn., Artech House (2005).

47. T. H. Taminiau, F. D. Stefani, and N. F. van Hulst, Opt. Express 16, 10858 (2008).

48. S. Tsujino, P. Beaud, E. Kirk, T. Vogel, H. Sehr, J. Gobrecht, and A. Wrulich, Appl. Phys. Lett. 92, 193501 (2008).

49. S. Tsujino, F. le Pimpec, J. Raabe, M. Buess, M. Dehler, E. Kirk, J. Gobrecht, and A. Wrulich, Appl. Phys. Lett. 94, 093508 (2009).
50. A. Vial, A. S. Grimault, D. Macías, D. Barchiesi, and M. Lamy de la Chapell, Phys. Rev. B 71, 085416 (2005).

51. J. L. Volakis, A. Chatterjee, and L. C. Kempel, Finite Element Method for Electromagnetics, 1st edn., IEEE/OUP Series on Electromagnetic Wave Theory, IEEE, 445 Hoes Lane, Piscataway, New Jersey (1998).

52. O. Zinke and H. Brunswig, Hochfrequenztechnik 1, 6th edn., Springer Verlag, Berlin-Heidelberg, New York (1999), Vol. 1.

Received: 5 October 2010. Accepted: 28 October 2010.

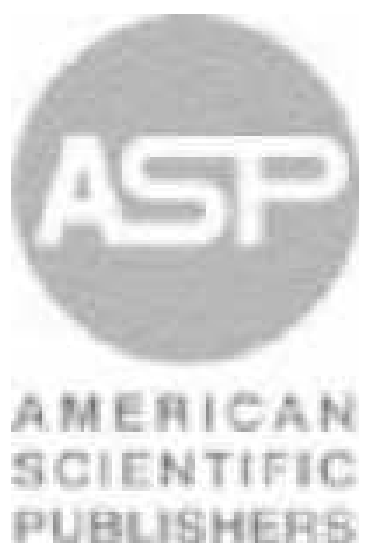

